Electronic Journal of Statistics

Vol. 15 (2021) 6429-6460

ISSN: 1935-7524

https://doi.org/10.1214/21-EJS1949

\title{
Testing marginal symmetry of digital noise images through the perimeter of excursion sets*
}

\author{
Mariem Abaach \\ MAP5 UMR CNRS 8145, Université de Paris, \\ 45 rue des Saints-Pères, 75006 Paris, France \\ e-mail: mariem.abaach@u-paris.fr \\ Hermine Biermé \\ LMA UMR CNRS 7348, Université de Poitiers, \\ 11 bd Marie et Pierre Curie, 86962 Chasseneuil, France \\ e-mail: hermine.bierme@math.univ-poitiers.fr
}

and

Elena Di Bernardino

Laboratoire J.A. Dieudonné, UMR CNRS 7351,

Université Côte d'Azur, Nice, France

e-mail: Elena.Di_bernardino@unice.fr

\begin{abstract}
In this paper we consider digital images for which the pixels values are given by a sequence of independent and identically distributed variables within an observation window. We proceed to the construction of an unbiased estimator for the perimeter without border effects. The study of the first and second moments of the perimeter allows to prove autonormalised asymptotic normality results with an explicit covariance matrix consistently estimated. Theses Central Limit Theorems permit to built a consistent and empirical accessible test statistic to test the symmetry of the marginal distribution. Finally the asymptotic perimeter behaviour in large threshold limit regime is also explored. Several numerical studies are provided to illustrate the proposed testing procedures.
\end{abstract}

MSC2020 subject classifications: Primary 60G60, 60D05; secondary 62F03, 60G10.

Keywords and phrases: Binary image, Crossings, Excursion sets, Test of symmetry, Threshold procedure.

Received February 2021.

*This work has been partially supported by the project ANR MISTIC (ANR-19-CE400005) and by the French government, through the 3IA Côte d'Azur Investments in the Future project managed by the National Research Agency (ANR) with the reference number ANR19-P3IA-0002. 


\section{Introduction}

The stochastic modeling of images by spatial random fields allows to set up a convenient statistical framework for different issues in image processing for image denoising, pattern detection, segmentation or classification [29, 22, 12].

A particular interest directed towards the study of geometric attributes and features of objects has grown these last years. Roughly speaking the considered quantities are the surface area, perimeter and Euler characteristic, which is a topological invariant that is equal to the number of connected components minus the number of holes, in a black and white binary image obtained by thresholding a gray image at some fixed level. These quantities are related to intrinsic volumes and Minkowski functionals particularly studied in the integral and stochastic geometry [8, 32] or Lipschitz-Killing (LK) curvatures of excursion sets (see [33] for a formal introduction to the subject). Those functionals are robust and efficient shape descriptors that have been largely applied to a variety of domains: cosmology (e.g. the morphological analysis of cosmic microwave background $[31,27,1]$ ), astrophysics (e.g. modeling galaxy formation [18]), military (e.g. mine field detection, [26]) medicine (e.g. brain imaging, [17], study of synthesized 2D digital mammograms, [4, 13]).

Very important results have been obtained in the framework of smooth stationary random fields, especially for Gaussian related fields (see [2]). In this framework, the theoretical means of LK curvatures are explicitly computed with respect to the parameters of the fields $[35,34,3]$ and can be efficiently estimated from images [4]. The consistent estimation of related variances had been recently studied in [13]. Moreover, Central Limit Theorems (CLT) have been proven for Gaussian fields $[16,23]$ that paved the road for statistical hypothesis tests. Several extensions for non-Gaussian fields, namely shot noise random fields are also available for means of LK curvatures [24] and associated CLT's [25]. However the framework of smooth fields, indexed by continuous space variables, is not appropriate with the discrete framework induced by digital images. Let us remark that links with discrete setting have been recently explored in [5].

In this paper we consider this discrete framework for digital images for which white noise is well defined. More precisely, we assume that pixels values are given by $\left(X_{i, j}\right)_{i, j \geq 1}$ a sequence of independent and identically distributed (i.i.d.) variables within an observation window $S$. We have in mind the residuals of a denoising procedure or a linear regression, or the difference between two images as for brain activities study. Therefore, we are interested to test the natural symmetry hypothesis, that is to know whether $X_{i, j}$ is drawn from a symmetrical distribution. This assumption of marginal distribution symmetry is called here the null hypothesis $H_{0}$ and includes Gaussian or Student distributions. Being able to formally test for symmetry hypotheses is an important topic in many fields since this basic assumption contains important information regarding the underlying model, that would allow to validate it before further investigation.

This problem has been largely considered in the literature for i.i.d. sample, 
especially due to its importance for time series analysis. Different approaches to construct a symmetry test include the use of empirical distribution for known center of symmetry [7], the characteristic symmetry function and study of its behavior to know if the distribution is symmetric or not [9] with unknown center. Other tests rely on the study of the skewness of the distribution function as initiated by [19]; see [28] for a free-distribution test based on Bonferroni's measure. For an overview and comparison of the existing methods we refer to [30] and [21].

In our original approach we attempt to use our digital image setting and the geometrical features of its excursion sets. More precisely, for a given threshold $t \in \mathbb{R}$ we consider the binary image given by the i.i.d. Bernoulli variables $\left(\mathbb{1}_{\left\{X_{i, j} \geq t\right\}}\right)_{i, j \geq 1}$, coloring black pixels for values equal to one and considering the area of black components as well as its perimeter inside a given domain. This allows us to build an unbiased estimator of the perimeter, without border effects. This framework is close from the one used by [15] to set up a goodness of fit test for complete spatial randomness. In the aforementioned paper the authors consider the counting process obtained from a homogeneous Poisson point process such that the observed values $\left(X_{i, j}\right)$ are i.i.d. Poisson random variables. They obtain explicit expectation and covariance matrix for the three Lipschitz-Killing curvatures at a fixed level as well as CLT to build their test. In the present paper, we focus on the perimeter and obtain a multivariate CLT with respect to several threshold levels with an explicit covariance matrix, under a general i.i.d. assumption, in a dense tiling framework. The choice of our unbiased perimeter estimator permits us to preserve the symmetry of the distribution, in contrast with perimeter with border. This lead us to construct the statistical test of symmetry, by considering the behavior of the ratio between two specific thresholds. We also explore the asymptotic in large threshold limit regime and obtain the equivalence between the asymptotic behavior of the expectation value and the variance establishing a joint auto-normalized Central Limit Theorem for dense tiling and large threshold limit regime in a white noise framework, similarly to [11] for crossings in dimension 1.

The paper is organized as follows. In Section 2 we introduce our mathematical framework for binary images and we proceed to the construction of an unbiased estimator for the associated perimeter. Then, in Section 3 we study the first and second moments of the perimeter, which allows us to prove asymptotic normality results with an explicit covariance matrix, for which we consider several consistent estimators. The construction of our consistent symmetry test via empirical accessible test statistics is proposed in Section 4. Numerical evaluations are presented in Section 5. The case of large threshold values is investigated in Section 6, that yields to a new a central limit theorem and test statistics illustrated with numerical comparisons. Finally, we postpone the technical proofs to Section 7, and additional numerical evaluations to the Appendix. 


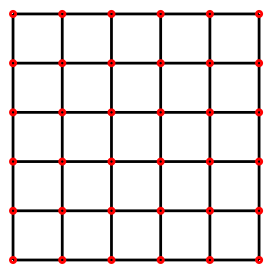

FIG 1. Tilling with squares for $m=5$ and associated vertices (red circles).
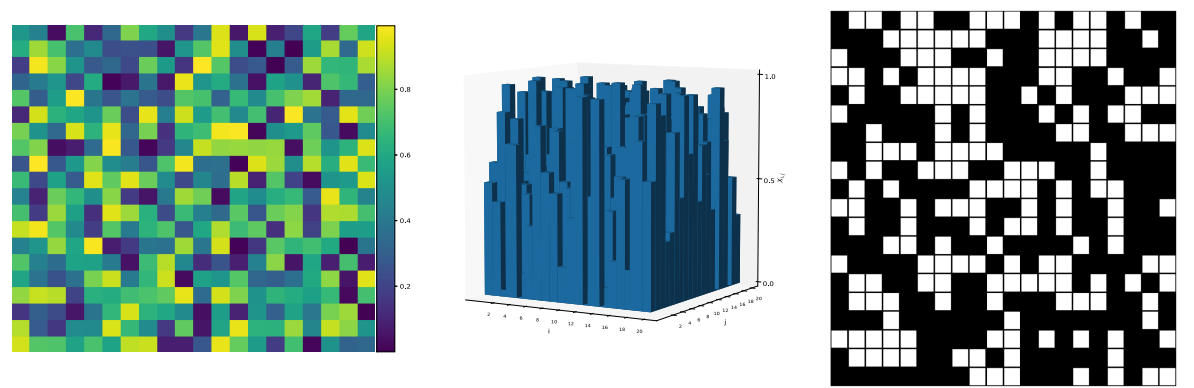

FIG 2. Left and center panels: Image of size $(20 \times 20)$ realization of a Uniform white noise model. Right panel: Obtained binary image for $t=0.5$.

\section{Mathematical framework}

\subsection{Construction of the binary image}

Square tiling Let $m$ be an integer with $m \geq 2$, without loss of generality, we consider our observation window as the unit square $S=[0,1]^{2}$ and we divide our window into $m^{2}$ pairwise disjoint squares. This provides a regular tiling of $S$ with squares of "size" (side length) $1 / m$, i.e.,

$$
C_{i, j}^{(m)}:=\left[\frac{i-1}{m}, \frac{i}{m}\right] \times\left[\frac{j-1}{m}, \frac{j}{m}\right], \text { for } i, j \in\{1, \ldots, m\} .
$$

The $C_{i, j}^{(m)}$ will be referred to as cells. We denote by $\mathcal{E}_{m}$ the set of edges in $\stackrel{\circ}{S}=(0,1)^{2}$, each $w \in \mathcal{E}_{m}$ is a segment of length $1 / m$.

Discrete setting Using the previous square tiling, we write $S=\underset{1 \leq i, j \leq m}{\cup} C_{i, j}^{m}$. Let $\left(X_{i, j}\right)_{1 \leq i, j \leq m}$ be a sequence of random variables defined over the same probability space $(\Omega, \mathcal{A}, \mathbb{P})$, that are independent and identically distributed. Each pixel value $X_{i, j}$ is associated to the cell $C_{i, j}^{(m)}$. In Figure $2, X_{i, j} \sim \mathcal{U}(0,1)$ and $m=20$.

Let us consider a threshold parameter $t \in \mathbb{R}$. In order to create the associated binary image, we introduce a random $m \times m$-matrix $Z(t)=\left(Z_{i, j}(t)\right)_{1 \leq i, j \leq m}$, where 


$$
Z_{i, j}^{(m)}(t):=\mathbb{1}_{\left\{X_{i, j} \geq t\right\}}, \text { for } i, j \in\{1, \ldots, m\} .
$$

Each cell $C_{i, j}^{(m)}$ is associated to black or white according to whether $Z_{i, j}(t)=0$ or $Z_{i, j}(t)=1$. Then, $Z_{i, j}(t)$ follows a binomial distribution of parameter $\left(1, p_{t}\right)$, where

$$
p_{t}:=\mathbb{P}\left(Z_{i, j}(t)=1\right)=\mathbb{P}\left(X_{i, j} \geq t\right)=1-F\left(t^{-}\right),
$$

with $F$ the cumulative distribution function associated to $X_{i, j}$.

\subsection{Perimeter of a binary image}

Let $Z=Z(t)$ be the binary image $Z$ at that given threshold $t \in \mathbb{R}$. Following the approach presented in [5], for each edge $w \in \mathcal{E}_{m}$, we aim to know if $w$ contributes to the perimeter of the black component of $Z$. Let $w$ be a horizontal edge of the form $w=\left(\frac{l-1}{m}, \frac{l}{m}\right) \times\left\{\frac{k-1}{m}\right\}, w$ belongs to both cells $C_{l, k-1}$ and $C_{l, k}$, which means that $w$ is a contribution to the perimeter if $Z_{l-1, k} \neq Z_{l, k}$. Following this consideration, one can consider

$$
f_{1}^{(t)}(l, k)=\mathbb{1}_{\left\{\left(Z_{l, k-1}(t)=0 \cap Z_{l, k}(t)=1\right) \cup\left(Z_{l, k-1}(t)=1 \cap Z_{l, k}(t)=0\right)\right\}}
$$

to count the horizontal contributions and

$$
f_{2}^{(t)}(k, l)=\mathbb{1}_{\left\{\left(Z_{k-1, l}(t)=0 \cap Z_{k, l}(t)=1\right) \cup\left(Z_{k-1, l}(t)=1 \cap Z_{k, l}(t)=0\right)\right\}}
$$

to count the vertical ones.

Definition 2.1 (Perimeter and scaled perimeter of a binary image). We denote by $\mathcal{P}_{m}^{(1)}(t)=\sum_{l=1}^{m} \sum_{k=2}^{m} f_{1}^{(t)}(l, k)$ the sum of all horizontal contributions and $\mathcal{P}_{m}^{(2)}(t)=\sum_{l=1}^{m} \sum_{k=2}^{m} f_{2}^{(\bar{t})}(k, l)$ the sum of the vertical ones, then, the perimeter and scaled perimeter are given by

$$
\mathcal{P}_{m}(t):=\mathcal{P}_{m}^{(1)}(t)+\mathcal{P}_{m}^{(2)}(t), \quad \quad \check{\mathcal{P}}_{m}(t):=\frac{1}{m^{2}}\left(\mathcal{P}_{m}^{(1)}(t)+\mathcal{P}_{m}^{(2)}(t)\right)
$$

An equivalent way to compute the perimeter is by considering, for each edge $w$, the maximal and minimal values on the two sides of $w$. Then, the perimeter is equivalently given by

$$
\mathcal{P}_{m}(t):=\sum_{w \in \mathcal{E}_{m}}\left(f_{+}^{(t)}(w)-f_{-}^{(t)}(w)\right)
$$

where $f_{+}^{(t)}(w)=\max \left(Z_{l, k-1}(t), Z_{l, k}(t)\right)$ and $f_{-}^{(t)}(w)=\min \left(Z_{l, k-1}(t), Z_{l, k}(t)\right)$ for $w$ the common edge between $C_{l, k-1}$ and $C_{l, k}$. The interested reader is referred to [5]. The only edges $w \in \mathcal{E}_{m}$ that contribute to the computation are those for which $f_{+}^{(t)}(w)=1$ and $f_{-}^{(t)}(w)=0$ which are exactly the edges belonging to the perimeter intersected with the interior of our observation window. 


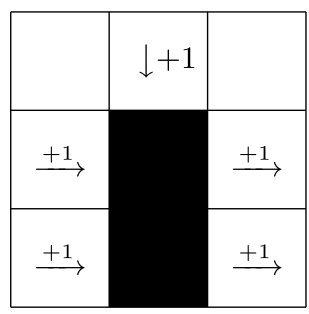

FIG 3. Computation of the perimeter of a binary image with $m=3$. Here $\mathcal{P}_{3}=5$.

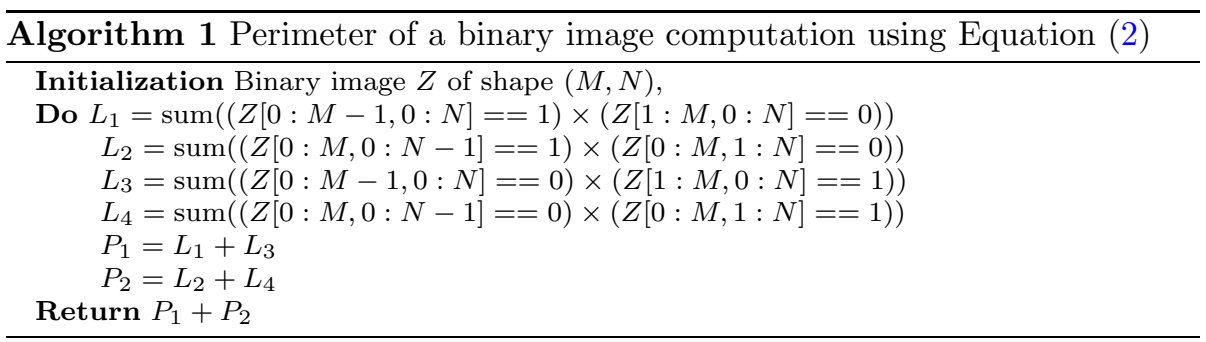

Remark 1. Notice that the proposed method in Definition 2.1 (see Equation (2)) does not take into account the contribution of the edges that belongs to the frontier of the observation window $S$. Indeed, in Algorithm 1, the border cells can only contribute to the perimeter with one or two edges contrary to the other cells that can have up to four edges contributing to the perimeter. In this sense this method is unbiased in comparison with [15]'s technique. In the aforementioned paper, the authors introduce a white artificial frontier around the image and then, compute the perimeter of the extended new image (white frontier included), making all cells contributing to the perimeter have a four edge contribution.

\section{Statistics of the perimeter of a binary image}

\subsection{Moments and asymptotic normality}

In this section we investigate the first and second moments of the perimeter. This preliminary study will be useful to state our multivariate Central Limit Theorem (see Theorem 3.3 below).

Proposition 3.1 (First moment of the perimeter). The expected value of the scaled perimeter in (1) is given by

$$
\mathbb{E}\left(\check{\mathcal{P}}_{m}(t)\right)=4 p_{t}\left(1-p_{t}\right)\left(1-\frac{1}{m}\right):=\mu_{\check{\mathcal{P}}}\left(p_{t}, m\right) .
$$

The proof of Proposition 3.1 is postponed to Section 7. 
Remark 2. Assume that $X_{i, j}$ is drawn from a symmetrical continuous distribution of axis $\theta \in \mathbb{R}$, i.e $p_{\theta-t}=1-p_{\theta+t}, \forall t \in \mathbb{R}$. Then, $\mu_{\check{\mathcal{P}}}\left(p_{\theta-t}, m\right)=\mu_{\check{\mathcal{P}}}\left(p_{\theta+t}, m\right)$ and therefore, $t \mapsto \mu_{\check{\mathcal{P}}}\left(p_{t}, m\right)$ has an axis of symmetry on $\theta$. This implies that the ratio $\left(\check{\mathcal{P}}_{m}(\theta-t)\right) /\left(\tilde{\mathcal{P}}_{m}(\theta+t)\right)$ would be distributed around 1 in the case of symmetry. This consideration will be crucial for the proposed symmetry testing procedure in Section 4.

We now study the second moment of the perimeter of the considered binary image.

Proposition 3.2 (Covariance between perimeters at two thresholds). Let $t, s$ $\in \mathbb{R}$ be two given thresholds. The covariance between the scaled perimeter taken at levels $t$ and $s$ is given by

$$
\begin{aligned}
\operatorname{Cov}\left(\check{\mathcal{P}}_{m}(t), \check{\mathcal{P}}_{m}(s)\right)= & \frac{4}{m^{4}} p_{\max (t, s)}\left(1-p_{\min (t, s)}\right)\left(\left(4 m^{2}-7 m+2\right)\right. \\
& -2 p_{\min (t, s)}\left(1-p_{\max (t, s)}\right)\left(7 m^{2}-13 m+4\right) \\
& \left.+2\left(p_{\min (t, s)}-p_{\max (t, s)}\right)\left(3 m^{2}-6 m+2\right)\right) .
\end{aligned}
$$

Taking $t=s$, we obtain the following variance formula

$$
\begin{aligned}
\operatorname{Var}\left(\check{\mathcal{P}}_{m}(t)\right) & =\frac{4}{m^{4}} p_{t}\left(1-p_{t}\right)\left(\left(4 m^{2}-7 m+2\right)-2 p_{t}\left(1-p_{t}\right)\left(7 m^{2}-13 m+4\right)\right) \\
& :=\sigma_{\check{\mathcal{P}}}^{2}(t, m) .
\end{aligned}
$$

The proof of Proposition 3.2 is postponed to Section 7. If we add in Proposition 3.2 the bias induced by the boundary of the window $S$ to the variance computation, we obtain the same results as those provided in [15] (see Theorem 4.1).

Beyond Propositions 3.1 and 3.2, we can prove the multivariate asymptotic normality as $m \rightarrow \infty$ of our geometrical feature for a given vector of thresholds.

Theorem 3.3 (Multivariate CLT for $r$-thresholds). Let $r$ be a positive integer, $m \geq 2$ and $t_{1}, \ldots, t_{r} \in \mathbb{R}$, then,

$$
m\left(\left(\begin{array}{c}
\check{\mathcal{P}}_{m}\left(t_{1}\right) \\
\check{\mathcal{P}}_{m}\left(t_{2}\right) \\
\vdots \\
\check{\mathcal{P}}_{m}\left(t_{r}\right)
\end{array}\right)-\left(\begin{array}{c}
\mathbb{E}\left(\check{\mathcal{P}}_{m}\left(t_{1}\right)\right) \\
\mathbb{E}\left(\check{\mathcal{P}}_{m}\left(t_{2}\right)\right) \\
\vdots \\
\mathbb{E}\left(\check{\mathcal{P}}_{m}\left(t_{r}\right)\right)
\end{array}\right)\right) \underset{m \rightarrow \infty}{\stackrel{d}{\longrightarrow}} \mathcal{N}\left(0, \Sigma_{r}^{\star}\right)
$$

where $\stackrel{d}{\rightarrow}$ holds for the convergence in distribution and $\mathcal{N}\left(0, \Sigma_{r}^{\star}\right)$ for the $r$ dimensional centered Gaussian distribution with covariance matrix $\Sigma_{r}^{\star}$ given by

$$
\begin{aligned}
\Sigma_{r}^{\star}(i, j):= & 4 p_{\max \left(t_{i}, t_{j}\right)}\left(1-p_{\min \left(t_{i}, t_{j}\right)}\right)\left(4-14 p_{\min \left(t_{i}, t_{j}\right)}\left(1-p_{\max \left(t_{i}, t_{j}\right)}\right)\right. \\
& \left.+6\left(p_{\min \left(t_{i}, t_{j}\right)}-p_{\max \left(t_{i}, t_{j}\right)}\right)\right) .
\end{aligned}
$$


The proof of Theorem 3.3 is postponed to Section 7. Furthermore, some numerical illustrations of Theorem 3.3 in the case $r=2$ can be found in Appendix A. Finally, as can be seen in (5), the last theoretical result that will be needed for the construction of an empirical accessible test statistic is an estimation of $p_{t}$. This will be discussed in the next section.

\subsection{Two consistent estimators for $p_{t}$.}

In the following, we will present two estimators and study their consistency properties. In this section, for the sake of clarity, we denote $p(t):=p_{t}, \forall t \in \mathbb{R}$.

One way to estimate $p(t)$ is by considering the area of the excursion set. Another idea for the estimation of $p(t)$ is built on the relationship that exists between the expectation value of the perimeter at a level $t$ and $p(t)$ (see Equation $(3))$ :

$$
\mathbb{E}\left(\frac{\check{\mathcal{P}}_{m}(t)}{\left(1-\frac{1}{m}\right)}\right)=4 p(t)(1-p(t))
$$

Definition 3.4 (Area and perimeter based estimator for $p(t)$ ). Given a threshold $t \in \mathbb{R}$, we define

$$
\widehat{p}_{m}^{\mathcal{A}}(t)=\frac{1}{m^{2}} \sum_{i, j=1}^{m} Z_{i, j}(t) .
$$

Besides, we partition the image $S$ in $m^{2} / 4$ sub-images $S_{2}^{i}$ of size $(2 \times 2)$. We denote by $\check{\mathcal{P}}_{2}^{i}(t)$ the value of the scaled perimeter for each sub-image. Let $\bar{S}_{m}(t):=\frac{4}{m^{2}} \sum_{i} \check{\mathcal{P}}_{2}^{i}(t)$ and the continuous mapping $g:[0,1] \rightarrow \mathbb{R}$ defined by

$$
x \mapsto g(x)= \begin{cases}\frac{1}{2}(1-\sqrt{1-2 x}) & \text { if } x<\frac{1}{2}, \\ \frac{1}{2} & \text { else. }\end{cases}
$$

Then, for $t>\theta$ with $\theta$ the median value of the distribution, we define the perimeter based estimator of $p(t)$ as

$$
\widehat{p}_{m}^{\mathcal{P}}(t):=g\left(\bar{S}_{m}(t)\right)
$$

Proposition 3.5 (Asymptotic normality for the proposed estimators for $p(t)$ ). Let $\widehat{p}_{m}^{\mathcal{A}}(t)$ and $\widehat{p}_{m}^{\mathcal{P}}(t)$ as in Definition 3.4. Then, it holds that

$$
\widehat{p}_{m}^{\mathcal{A}}(t) \underset{m \rightarrow \infty}{\stackrel{a . s}{\longrightarrow}} p(t) \quad \text { and } \quad m\left(\widehat{p}_{m}^{\mathcal{A}}(t)-p(t)\right) \underset{m \rightarrow \infty}{\stackrel{d}{\longrightarrow}} \mathcal{N}(0, p(t)(1-p(t))) .
$$

Similarly, it holds

$$
\widehat{p}_{m}^{\mathcal{P}}(t) \underset{m \rightarrow \infty}{\stackrel{a . s}{\longrightarrow}} p(t)
$$

and

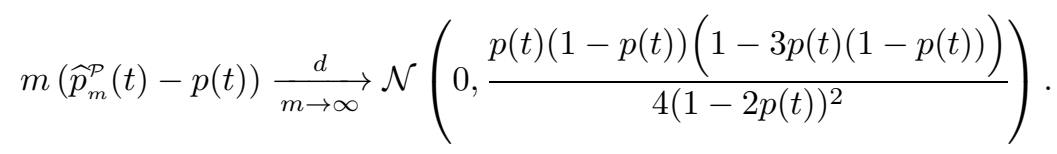


The proof of Proposition 3.5 is postponed to Section 7 .

Remark 3. It is also possible to estimate $p(t)$ for $t<\theta$ by considering the continuous mapping $h:[0,1] \rightarrow \mathbb{R}, x \mapsto h(x)= \begin{cases}\frac{1}{2}(1+\sqrt{1-2 x}) & \text { if } x<\frac{1}{2}, \\ \frac{1}{2} & \text { else, }\end{cases}$ and applying the same procedure.

\section{Perimeter based symmetry test}

As previously discussed in Remark 2, we now aim to build a test using the specific structure of the mean perimeter in the case of symmetry. Let $I=\left\{x \in \mathbb{R}, 0<p(x)<\frac{1}{2}\right\}$ and $I_{\theta}=I-\theta$, it follows that

$$
\forall t \in I_{\theta}, 0<p(\theta+t)<\frac{1}{2} .
$$

A test statistic Following the idea in [4] we propose a method to test the symmetry of the marginal distribution marginal of the field at equidistant levels from the median.

Let us consider the null hypothesis for $t \in I_{\theta}$

$$
H_{0}(t): p_{\theta-t}=1-p_{\theta+t},
$$

where $\theta$ is the median value of the distribution. Let us first present a normality asymptotic result in the general case and a second result under the null hypothesis.

Proposition 4.1 (Ratio between perimeters at two thresholds). Let $t_{1} \in \mathbb{R}$ and $t_{2} \in I$ with $t_{1}<t_{2}$, and $\check{\mathcal{P}}_{m}(\cdot)$ be the scaled perimeter in Definition 2.1 computed for each threshold. Then, it holds that,

$$
m\left(\frac{\check{\mathcal{P}}_{m}\left(t_{1}\right)}{\check{\mathcal{P}}_{m}\left(t_{2}\right)}-\frac{\mu_{\check{\mathcal{P}}_{m}}\left(t_{1}\right)}{\mu_{\check{\mathcal{P}}_{m}}\left(t_{2}\right)}\right) \underset{m \rightarrow \infty}{\stackrel{d}{\longrightarrow}} \mathcal{N}\left(0, \widetilde{\sigma}^{2}\left(t_{1}, t_{2}\right)\right)
$$

with $\widetilde{\sigma}^{2}\left(t_{1}, t_{2}\right)=\frac{p_{t_{1}}\left(1-p_{t_{1}}\right)}{\left(p_{t_{2}}\left(1-p_{t_{2}}\right)\right)^{3}}\left(p_{t_{2}}\left(1-p_{t_{2}}\right)-2 p_{t_{2}}\left(1-p_{t_{1}}\right)-3 p_{t_{2}}\left(p_{t_{1}}-p_{t_{2}}\right)\right.$ $\left.\left(1-p_{t_{1}}\right)+p_{t_{1}}\left(1-p_{t_{1}}\right)\right)$.

Let $t \in \mathcal{I}_{\theta}$ and $R_{m, \theta}(t):=\frac{\check{\mathcal{P}}_{m}(\theta-t)}{\check{\mathcal{P}}_{m}(\theta+t)}$. Then, under $H_{0}(t)$, it holds that

$$
m\left(R_{m, \theta}(t)-1\right) \underset{m \rightarrow \infty}{\stackrel{d, H_{0}}{\longrightarrow}} \mathcal{N}\left(0, \sigma^{2}(\theta+t)\right),
$$

with $\sigma^{2}(\theta+t)=\frac{\left(2 p_{\theta+t}-1\right)\left(3 p_{\theta+t}-2\right)}{p_{\theta+t}\left(1-p_{\theta+t}\right)^{2}}$.

We postpone the proof of Proposition 4.1 in Section 7 . 


\subsection{Proposed test with asymptotic level $\alpha$}

Let $\alpha \in(0,1)$ and $q_{1-\alpha / 2}$ such that $\mathbb{P}\left(\mathcal{N}(0,1) \leq q_{1-\alpha / 2}\right)=1-\alpha / 2$. Then, using Proposition 4.1, we define the statistic of the test with asymptotic level $\alpha$,

$$
\phi_{m}^{\mathcal{P}}(\sigma)=\mathbb{1}\left\{\left|\frac{m}{\sigma(\theta+t)}\left(R_{m, \theta}(t)-1\right)\right| \geq q_{1-\alpha / 2}\right\} .
$$

Firstly, we study the consistency of the proposed test statistic (see Proposition 4.2 below). Secondly, we focus on some estimation procedures for $\sigma(\theta+t)$ (see Section 4.2).

Let $t \in \mathcal{I}_{\theta}$ and $H_{1}(t)$ be the alternative hypothesis

$$
H_{1}(t): p_{\theta-t} \neq 1-p_{\theta+t} .
$$

Proposition 4.2 (Consistency of the proposed symmetry test). For $t \in \mathcal{I}_{\theta}$, it holds that $\mathbb{P}_{H_{1}(t)}\left(\phi_{m}^{\mathcal{P}}(\sigma)=1\right) \rightarrow 1$ for $m \rightarrow \infty$, with $\phi_{m}^{\mathcal{P}}(\sigma)$ as in Equation (8).

We postpone the proof of Proposition 4.2 in Section 7.

\subsection{Empirical accessible test statistic}

Notice that, if we take a consistent empirical estimator $\widehat{\sigma}_{m}(\theta+t)$ of the variance of the considered ratio at level $\theta+t$, then, we can consider the empirical accessible test statistic

$$
\phi_{m}^{\mathcal{P}}(\widehat{\sigma})=\mathbb{1}\left\{\left|\frac{m}{\widehat{\sigma}_{m}(\theta+t)}\left(R_{m, \theta}(t)-1\right)\right| \geq q_{1-\alpha / 2}\right\} .
$$

Using the estimators of $p(t)$ presented in Proposition 3.5, we can now construct estimators of the variance of the ratio to built $\phi_{m}^{\mathcal{P}}(\widehat{\sigma})$ in $(9)$.

Proposition 4.3 (Plug-in variance estimators). Let $\widehat{p}_{m}(\theta+t)$ be a consistent estimator of $p(\theta+t)$ (i.e $\widehat{p}_{m}(\theta+t) \rightarrow p(\theta+t)$, a.s.) and $\sigma(\theta+t)$ as in Proposition 4.1. We define

$$
\widehat{\sigma}_{m}(\theta+t):=\sqrt{\frac{\left(2 \widehat{p}_{m}(\theta+t)-1\right)\left(3 \widehat{p}_{m}(\theta+t)-2\right)}{\widehat{p}_{m}(\theta+t)\left(1-\widehat{p}_{m}(\theta+t)\right)^{2}}} .
$$

Then, $\widehat{\sigma}_{m}(\theta+t) \underset{m \rightarrow \infty}{\stackrel{a . s .}{\longrightarrow}} \sigma(\theta+t)$ and

$$
\frac{m}{\widehat{\sigma}_{m}(\theta+t)}\left(R_{m, \theta}(t)-1\right) \underset{m \rightarrow \infty}{\stackrel{d, H_{0}}{\longrightarrow}} \mathcal{N}(0,1)
$$

The proof of Proposition 4.3 is postponed to Section 7. 
Corollary 4.4. Using the same framework as in Proposition 4.3, we denote by $\widehat{\sigma}_{m}^{\mathcal{P}}(\theta+t)$ and $\widehat{\sigma}_{m}^{\mathcal{A}}(\theta+t)$ the plug-in estimators based on Equation (10) using respectively $\widehat{p}_{m}^{\mathcal{P}}(\theta+t)$ and $\widehat{p}_{m}^{\mathcal{A}}(\theta+t)$ as in Proposition 3.5. Then, $\widehat{\sigma}_{m}^{\mathcal{P}}(\theta+t)$ and $\widehat{\sigma}_{m}^{\mathcal{A}}(\theta+t)$ are two consistent estimators of $\sigma(\theta+t)$ and asymptotic normality in (11) holds for these two estimators.

Thus, from Corollary 4.4 and using Equation (9), we get two empirical accessible test statistics

$$
\phi_{m}^{\mathcal{P}}\left(\widehat{\sigma}^{\mathcal{P}}\right)=\mathbb{1}\left\{\left|\frac{m}{\hat{\sigma}_{m}^{\mathcal{P}}(\theta+t)}\left(R_{m, \theta}(t)-1\right)\right| \geq q_{1-\alpha / 2}\right\}
$$

and

$$
\phi_{m}^{\mathcal{P}}\left(\widehat{\sigma}^{\mathcal{A}}\right)=\mathbb{1}\left\{\left|\frac{m}{\widehat{\sigma}_{m}^{A}(\theta+t)}\left(R_{m, \theta}(t)-1\right)\right| \geq q_{1-\alpha / 2}\right\}
$$

\subsection{Comparison with Kolmogorov-Smirnov based estimator}

Remark that under $H_{0}(t)$, the cumulative distribution function $F$ satisfies $F(\theta-t)=1-F(\theta+t)$. Then, considering the empirical cumulative distribution function $t \mapsto \widehat{F}_{m}(t):=\frac{1}{m^{2}} \sum_{i, j} \mathbb{1}_{X_{i, j} \leq t}$, which is a robust estimator of $F$, one can build an alternative symmetry test. Let us note that $\widehat{F}_{m}\left(t^{-}\right)=1-\widehat{p}_{m}^{\mathcal{A}}(t)$ with $\widehat{p}_{m}^{\mathcal{A}}(t)$ as in Definition 3.4.

Proposition 4.5. Let $t \in(0, \infty)$. Under $H_{0}(t)$, it holds that

$$
m\left(\frac{1-\widehat{p}_{m}^{\mathcal{A}}(\theta-t)}{\widehat{p}_{m}^{\mathcal{A}}(\theta+t)}-1\right) \underset{m \rightarrow \infty}{\stackrel{d, H_{0}}{\longrightarrow}} \mathcal{N}\left(0,\left(\sigma^{k-s}\right)^{2}(\theta+t)\right),
$$

with $\left(\sigma^{k-s}\right)^{2}(\theta+t)=\frac{2}{p(\theta+t)}$.

The proof of Proposition 4.5 is postponed to Section 7 .

Let us denote

$$
\widehat{\sigma}_{m}^{\mathrm{k}-\mathrm{s}}(\theta+t)=\sqrt{\frac{2}{\widehat{p}_{m}^{\mathcal{A}}(\theta+t)}} \quad \text { and } \quad R_{m, \theta}^{\mathrm{k}-\mathrm{s}}(t):=\frac{1-\widehat{p}_{m}^{\mathcal{A}}(\theta-t)}{\widehat{p}_{m}^{\mathcal{A}}(\theta+t)} .
$$

One can provide an alternative Kolmogorov-Smirnov based test statistic with asymptotic level $\alpha$

$$
\phi_{m}^{\mathrm{k}-\mathrm{s}}\left(\widehat{\sigma}^{\mathrm{k}-\mathrm{s}}\right)=\mathbb{1}\left\{\left|\frac{m}{\widehat{\sigma}_{m}^{\mathrm{k}-\mathrm{s}(\theta+t)}}\left(R_{m, \theta}^{\mathrm{k}-\mathrm{s}}(t)-1\right)\right| \geq q_{1-\alpha / 2}\right\} .
$$

Remark 4. In particular for $s \in I$ we can prove that $\sigma^{2}(s)<\sigma_{\mathrm{k}-\mathrm{s}}^{2}(s)$, where $\sigma^{2}(s)$ is given by (7) and $\sigma_{\mathrm{k}-\mathrm{s}}^{2}(s)$ by (14), as shown in Figure 11 in Appendix $\mathrm{B}$. Then, the perimeter based test has a smaller variance than the alternative Kolmogorov-Smirnov one. 


\section{Some numerical studies for the proposed symmetry test}

In this section, we report simulation results for our symmetry test, for samples of 500 images of size $m \times m$, for $m=100,512,1024$ and different choices of threshold level. Firstly, we display the results for three symmetrical distributions (Gaussian, Student and Uniform distributions, see Figure 4) and three asymmetrical ones (Exponential, Chi-squared and Skew-normal distributions, see Figure 5).

For a better approximation of $\theta$, we use the mean estimator provided by the NumPy library, making use of the fact that for a symmetrical distribution it is equivalent to use the mean or median to characterize the symmetry of a distribution [i.e. a random variable $X$ is symmetrically distributed around $\theta$, the center of symmetry, if its distribution function $F$ satisfies $F(\theta-x)=1-F(\theta+$ $x)$ ], although, we decided to use the median estimator for $\theta$ in the alternative hypthesis. As we can appreciate in Figure 4, the test is able to successfully accept the $H_{0}$ hypothesis. However, the choice of the threshold is crucial in regard to the quality of the test. Unsurprisingly, for extreme thresholds, the test is less precise. We will focus on this important point in Section 6 below. Notice that the performances of our test statistic $\phi_{m}^{\mathcal{P}}$ with the two proposed estimators of the variance $\widehat{\sigma}^{\mathcal{A}}$ and $\widehat{\sigma}^{\mathcal{P}}$ (in red crosses and blue diamonds, respectively) seem to be globally similar. Furthermore, these satisfactory results are comparable to those obtained with the Kolmogorov-Smirnov based estimator in (15) (black stars).

Figure 5 numerically describes the good performance of our test $\phi_{m}^{\mathcal{P}}$ to distinguish $H_{1}(t)$ from $H_{0}(t)$, for $t \in \mathbb{R}_{+}^{\star}$, to test the alternative hypothesis, we choose to consider the median estimator to compute the value of $\theta$. We focus our study on three asymmetric distributional cases: Skew-normal distribution with probability density function given by $f(x)=2 \phi(x) \Phi(a x)$, for $x \in \mathbb{R}$, with $a \geq 0$, $\phi(\cdot)$ (resp. $\Phi(\cdot))$ the standard normal probability density function (resp. standard normal cumulative distribution function), Exponential distribution with parameter 1 and $\chi^{2}$ distribution with degree of freedom 1. Unsurprising, in the skew-normal case, we observe that when $a$ is larger the power of the test increases, indeed the smaller the parameter $a$, the less asymmetric the distribution will be. This behavior can be easily observed for the four chosen levels of skewness parameter $a=0.1,0.5,1,3$ in Figure 5 (from first to fourth panel).

Investigation of the ratio behavior in the spatially dependent framework In this section, we aim to investigate the behavior of the ratios previously constructed using the perimeter and Kolmogrov-Smirnov method in a dependent framework. Let recall some notations

$$
R_{m, \theta}(t)=\frac{\check{\mathcal{P}}_{m}(\theta-t)}{\check{\mathcal{P}}_{m}(\theta+t)} \quad \text { and } \quad R_{m, \theta}^{\mathrm{k}-\mathrm{s}}(t)=\frac{1-\widehat{p}_{m}^{\mathcal{A}}(\theta-t)}{\widehat{p}_{m}^{\mathcal{A}}(\theta+t)}
$$

with $\check{\mathcal{P}}_{m}(\cdot)$ as in Definition 2.1 and $\widehat{p}_{m}^{\mathcal{A}}(\cdot)$ as in Definition 3.4. 
Gaussian marginal distribution $\mathcal{N}(0,1)$
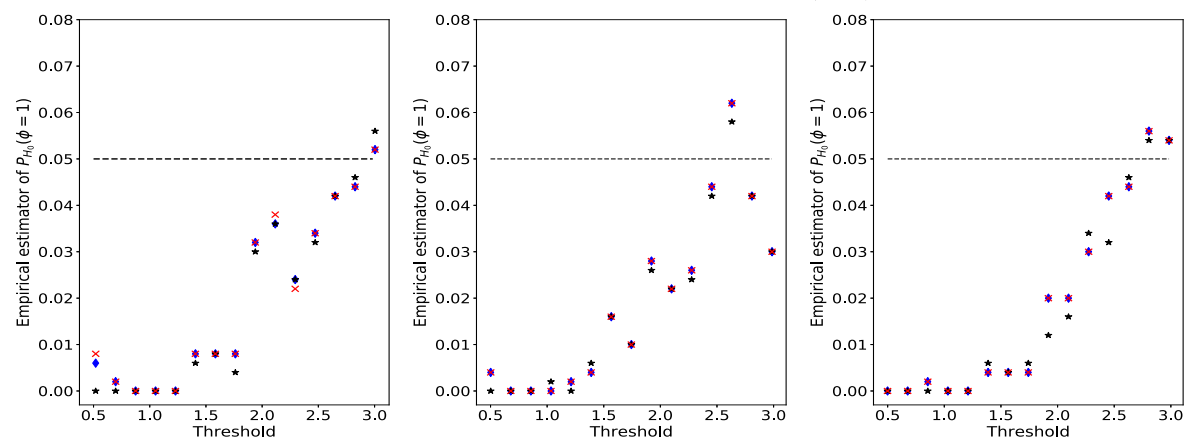

Student marginal distribution with $\nu=3$ degrees of freedom
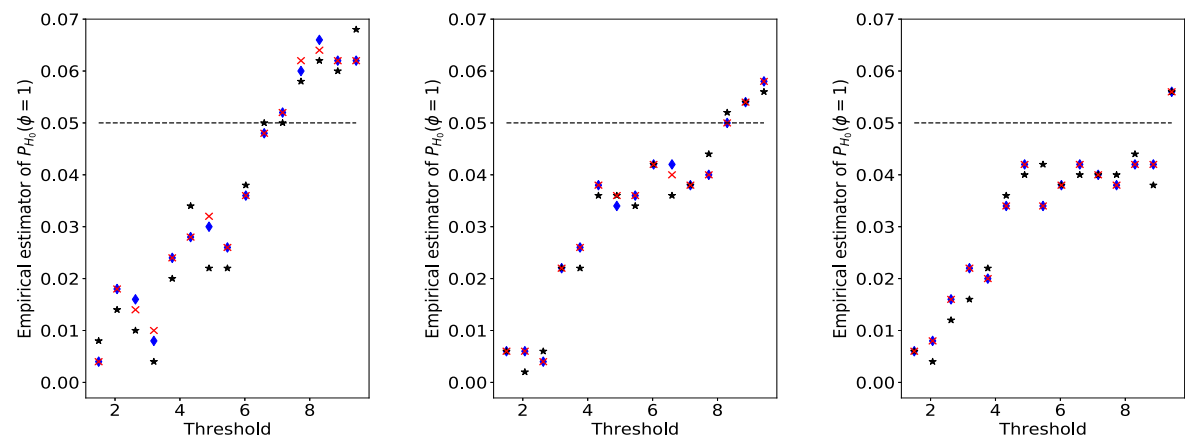

Uniform marginal distribution $\mathcal{U}(0,1)$
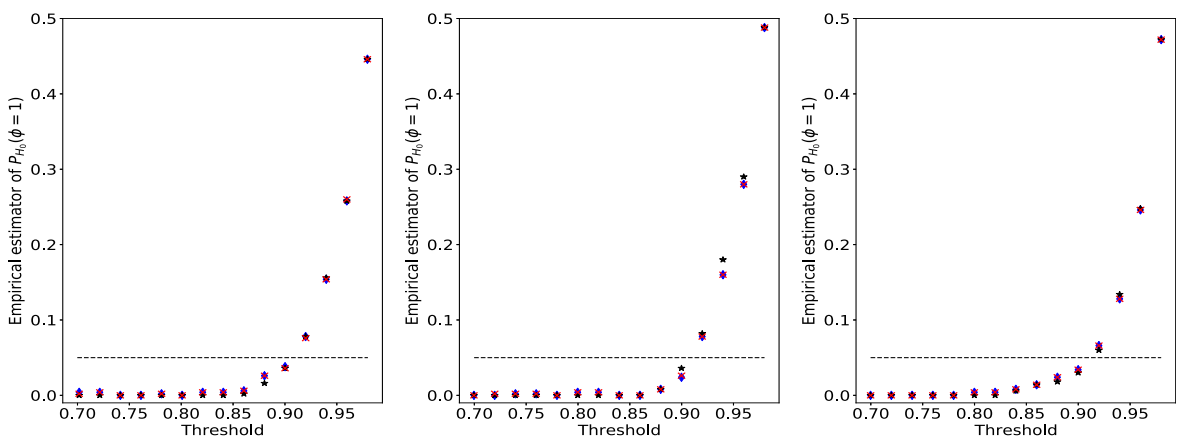

FIG 4. Under $H_{0}$ hypothesis. Average of the empirical values of $\mathbb{P}_{H_{0}(t)}\left(\phi_{m}^{\mathcal{P}}=1\right)$ for different thresholds t, for 500 Montecarlo simulations and for $m=100$ (left panels), 512 (center panels) and 1024 (right panels). We consider Gaussian marginal distribution $\mathcal{N}(0,1)$ (first row), Student marginal distribution with $\nu=3$ degrees of freedom (second row), Uniform marginal distribution $\mathcal{U}(0,1)$ (third row) and three different test statistics: $\phi_{m}^{\mathcal{P}}\left(\widehat{\sigma}^{\mathcal{P}}\right)$ as in (12) (blue diamonds), $\phi_{m}^{\mathcal{P}}\left(\widehat{\sigma}^{\mathcal{A}}\right)$ as in (13) (red crosses) and $\phi_{m}^{k-s}\left(\widehat{\sigma}^{k-s}\right)$ as in (15) (black stars). Threshold $\alpha=0.05$ is displayed by a dashed horizontal line.

In Figures 6 and 7 we evaluate for 500 Montecarlo simulations the average of the absolute error between the estimated ratio $R_{m, \theta}(t)$ and $R_{m, \theta}^{\mathrm{ks}}(t)$ and the 
Skew-normal, Exponential and Chi-squared marginal distributions
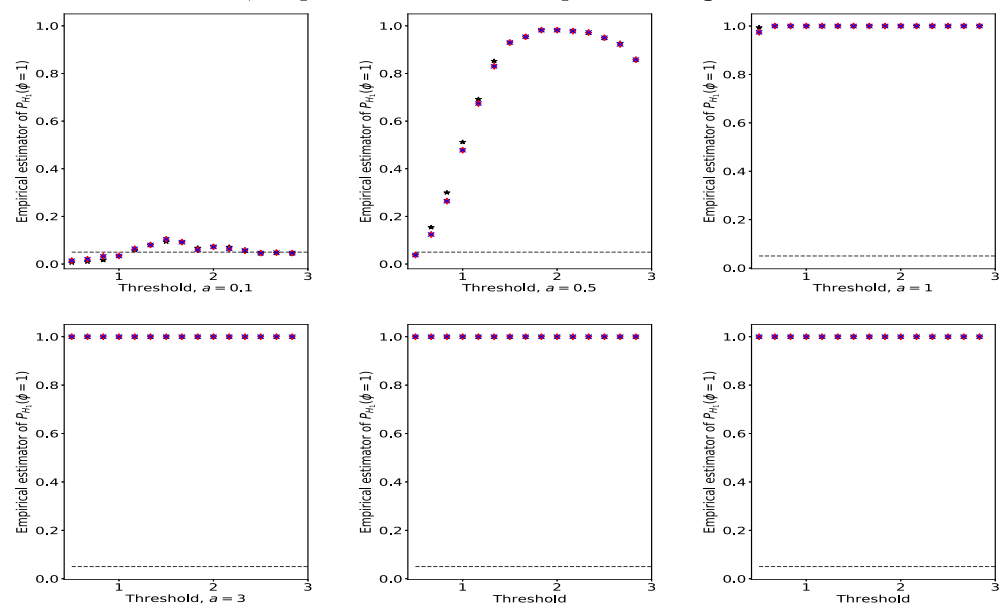

FIG 5. Under $H_{1}$ hypothesis. Average of the empirical values of $\mathbb{P}_{H_{1}(t)}\left(\phi_{m}^{\mathcal{P}}=1\right)$ for different thresholds $t$, for 500 Montecarlo simulations for $m=512$ with a skew-normal marginal distribution centered around its median value, with different choices of parameter $a=0.1,0.5,1,3$ (from first to fourth panel), $\operatorname{Exp}(1)-\theta$ distribution (fifth panel) and $\chi^{2}-\theta$ distribution of degree of freedom 1 (sixth panel), $\theta$ been the median value of the image. We consider different test statistics: $\phi_{m}^{\mathcal{P}}\left(\widehat{\sigma}^{\mathcal{P}}\right)$ as in (12) (blue crosses), $\phi_{m}^{\mathcal{P}}\left(\widehat{\sigma}^{\mathcal{A}}\right)$ as in (13) (red diamonds) and $\phi_{m}^{k-s}\left(\widehat{\sigma}^{k-s}\right)$ as in (15) (black stars). Threshold $\alpha=0.05$ is displayed by a dashed horizontal line.

targeted value 1 , i.e.,

$$
\widehat{\mathbb{E}}\left[\left|R_{m, \theta}(t)-1\right|\right] \quad \text { and } \quad \widehat{\mathbb{E}}\left[\left|R_{m, \theta}^{\mathrm{k}-\mathrm{s}}(t)-1\right|\right] .
$$

In Figure 6 we consider a smooth Gaussian random field $X$ of zero mean and unit variance of covariance structure given by $\rho(x)=\exp \left(-\kappa|| x \|_{2}^{2}\right.$ ) (under $H_{0}$ hypothesis), in Figure 7 a chi-squared field with degree of freedom given by $X^{2}$ (under $H_{1}$ hypothesis, see for instance Section 2.2.2 in [4]).

We can observe in these numerical studies, that the ratio constructed by using $R_{m, \theta}(t)$ performs slightly better than the one with $R_{m, \theta}^{\mathrm{k}-\mathrm{s}}$ in a dependent framework (for a small spatial dependence parameter $\kappa$, see right panels in Figures 6 and 7). This can be motivated by the fact that, in the considered dependent framework, the perimeter is a natural suitable candidate to get information about the underlying spatial structure and take into account the interconnection between the pixels.

Here we investigate the behavior of the empirical ratios and not of the associated test statistics to reduce the bias induced by the empirical estimation of unknown variances. These numerical investigations allow us to conjecture that, in a dependent framework, the geometrical feature of the perimeter contains important information about the field. A work is actually in progress in this direction. 

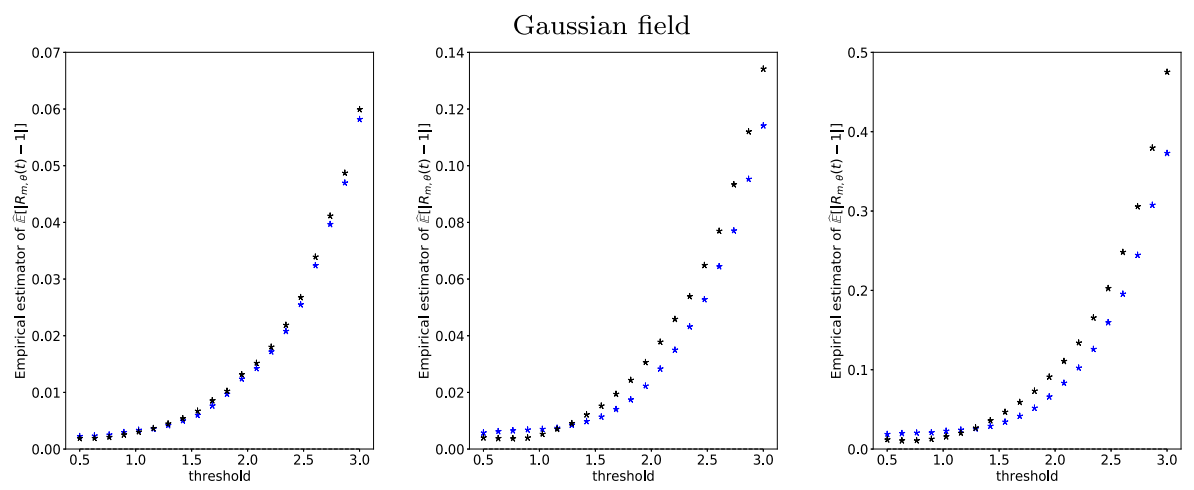

FIG 6. Under $H_{0}$ hypothesis. $\widehat{\mathbb{E}}\left[\left|R_{m, \theta}(t)-1\right|\right]$ (blue stars) and $\widehat{\mathbb{E}}\left[\left|R_{m, \theta}^{k-s}(t)-1\right|\right]$ (black stars), for different thresholds $t$, for 500 Montecarlo simulations and $m=512$. We consider a smooth stationnary Gaussian random field of covariance structure given by $\rho(x)=$ $\exp \left(-\kappa\|x\|_{2}^{2}\right), x \in \mathbb{R}^{2}$ for different values of $\kappa: 1$ (left panel), 0.1 (center panel) and 0.01 (right panel).

\section{Perimeter based symmetry test for large threshold and dense tiling}

Following the considerations in Section 5, we aim to improve the quality of the proposed test for large thresholds. As in [11], we firstly study the convergence of the ratio between the first two moments of the unscaled perimeter $\mathcal{P}_{m}$ (see Equations (3) and (4)). In Lemma 6.1 below, the expectation and the variance are proven to have the same order of magnitude for large threshold and dense tiling.

Lemma 6.1 (First two moments ratio for large threshold). Let $\mathcal{P}_{m}(t)$ as in (1) and $\mu_{\mathcal{P}}\left(p_{t}, m\right)$ and $\sigma_{\mathcal{P}}^{2}\left(p_{t}, m\right)$ the associated mean and variance. Let $\left(t_{m}\right)$ be a sequence of positive real numbers such that $p_{t_{m}} \rightarrow 0$ as $m \rightarrow \infty$, then,

$$
\frac{\sigma_{\mathcal{P}}^{2}\left(p_{t_{m}}, m\right)}{\mu_{\mathcal{P}}\left(p_{t_{m}}, m\right)} \underset{m \rightarrow \infty}{\longrightarrow} 4
$$

and furthermore, for $m$ large enough,

$$
0.5 \leq \frac{\sigma_{\mathcal{P}}^{2}\left(p_{t_{m}}, m\right)}{\mu_{\mathcal{P}}\left(p_{t_{m}}, m\right)} \leq 4
$$

The proof of Lemma 6.1 is postponed to Section 7. By using Lemma 6.1 we can now formulate a bivariate Central Limit Theorem for our geometrical feature for $m \rightarrow \infty$ and large thresholds.

Proposition 6.2 (Bivariate Central Limit Theorem for large threshold and dense tiling). Let $0<\gamma<2$ and $t_{(m, \gamma)}$ and $s_{(m, \gamma)}$ two points of continuity for the cumulative distribution function $F$, such that

$$
t_{(m, \gamma)}:=F^{-1}\left(\frac{1}{m^{\gamma}}\right) \quad \text { and } \quad s_{(m, \gamma)}:=F^{-1}\left(1-\frac{1}{m^{\gamma}}\right) .
$$



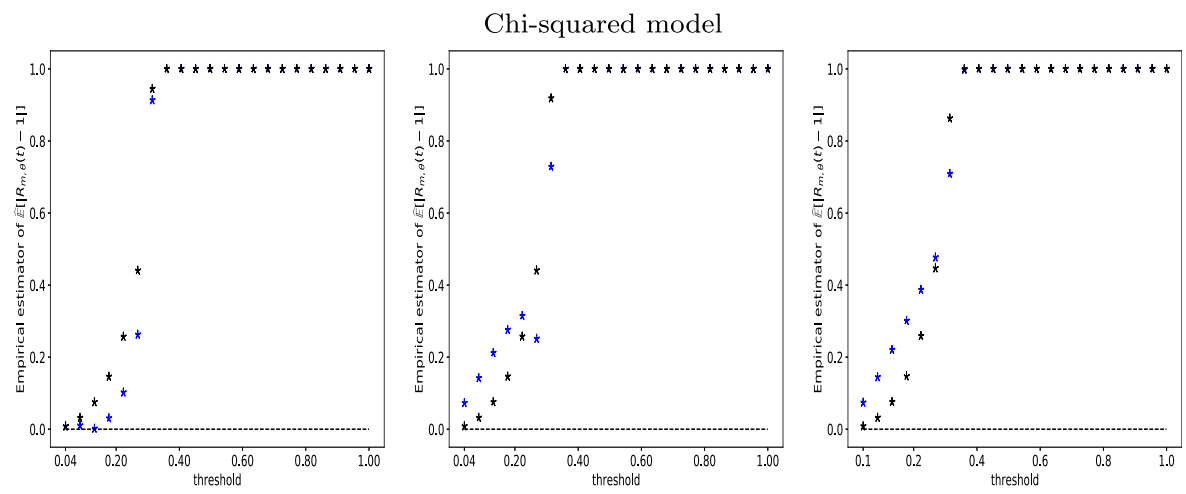

FIG 7. Under $H_{1}$ hypothesis. $\widehat{\mathbb{E}}\left[\left|R_{m, \theta}(t)-1\right|\right]$ (blue stars) and $\widehat{\mathbb{E}}\left[\left|R_{m, \theta}^{k-s}(t)-1\right|\right]$ (black stars), for different thresholds $t$, for 500 Montecarlo simulations and $m=512$. We consider a chi-square field with 1 degree of freedom, i.e., $\frac{1}{\sqrt{2}} X(t)^{2}-\theta$, with $X$ a centered with unit variance smooth stationnary Gaussian random field of covariance structure given by $\rho(x)=\exp \left(-\kappa\|x\|_{2}^{2}\right), x \in \mathbb{R}^{2}$ and $\theta$ the median value of $\frac{1}{\sqrt{2}} X(t)^{2}$, represented for different values of $\kappa: 1$ (left panel), 0.1 (center panel) and 0.01 (right panel).

Then, it holds that

$$
\sqrt{\frac{m^{2} \mathbb{E}\left(\check{\mathcal{P}}_{m}\left(t_{(m, \gamma)}\right)\right)}{4}}\left(\left(\begin{array}{c}
\frac{\check{\mathcal{P}}_{m}\left(t_{(m, \gamma)}\right)}{\mathbb{E}\left(\check{\mathcal{P}}_{m}\left(t_{(m, \gamma)}\right)\right)} \\
\frac{\check{\mathcal{P}}_{m}\left(s_{(m, \gamma)}\right)}{\mathbb{E}\left(\check{\mathcal{P}}_{m}\left(s_{(m, \gamma)}\right)\right)}
\end{array}\right)-\left(\begin{array}{l}
1 \\
1
\end{array}\right)\right) \underset{m \rightarrow \infty}{\stackrel{d}{\longrightarrow}} \mathcal{N}\left(\left(\begin{array}{l}
0 \\
0
\end{array}\right),\left(\begin{array}{ll}
1 & 0 \\
0 & 1
\end{array}\right)\right) .
$$

The proof of Proposition 6.2 is postponed to Section 7. Note that we have written $\mathbb{E}\left(\check{\mathcal{P}}_{m}\left(t_{(m, \gamma)}\right)\right)$ and $\mathbb{E}\left(\check{\mathcal{P}}_{m}\left(s_{(m, \gamma)}\right)\right)$ in $(16)$ for visual symmetry purposes although those two quantities are equal (see Equation (3)). Finally, we can easily derive the following result.

Corollary 6.3. Under assumptions of Proposition 6.2, it holds that

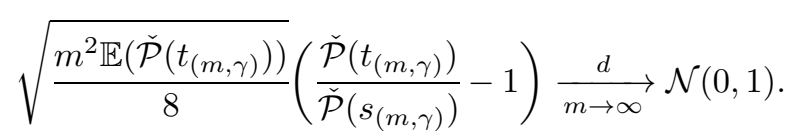

Furthermore,

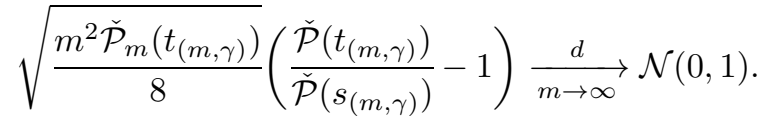

Equation (17) is obtained via the Delta method applied to Proposition 6.2. Finally, Equation (18) is given by using that, for $m \rightarrow \infty$, $\check{\mathcal{P}}_{m}\left(t_{(m, \gamma)}\right) / \mathbb{E}\left(\check{\mathcal{P}}_{m}\left(t_{(m, \gamma)}\right)\right) \stackrel{\mathbb{P}}{\rightarrow} 1$ and by applying the Slutsky Theorem. 
Gaussian marginal distribution $\mathcal{N}(0,1)$
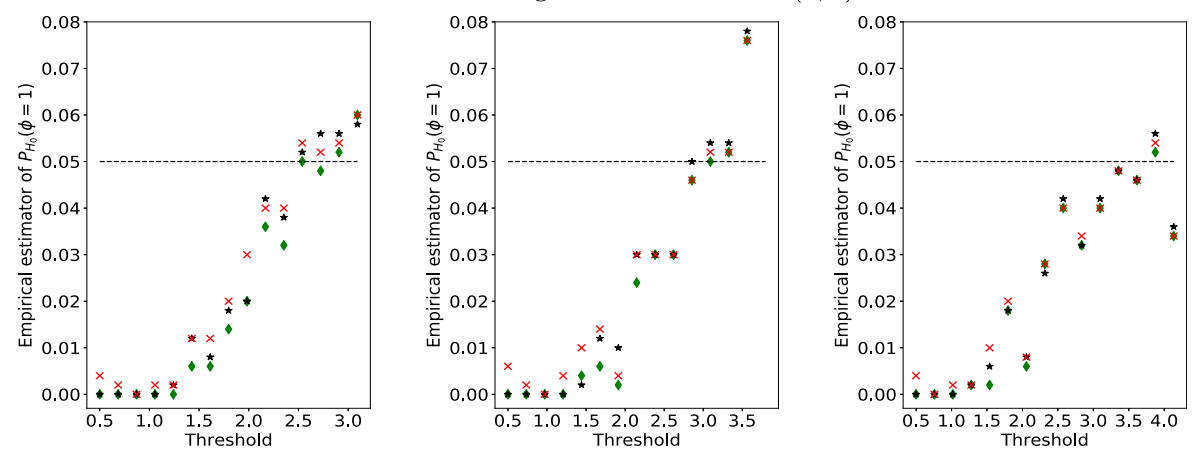

FIG 8. Under $H_{0}$ hypothesis. Average of the empirical values of $\mathbb{P}_{H_{0}(t)}\left(\phi_{m}^{\mathcal{P}}=1\right)$ for different thresholds $t_{m, \gamma}$, for 500 Montecarlo simulations, for $m=100$ (left panels), 512 (center panels) and 1024 (right panels). We consider a Gaussian marginal distribution $\mathcal{N}(0,1)$ and different test statistics: $\phi_{m}^{\mathcal{P}}\left(\widehat{\sigma}^{\mathcal{A}}\right)$ as in (13) (red stars), $\phi_{m}^{k-s}\left(\widehat{\sigma}^{k-s}\right)$ as in (15) (black stars) and $\phi_{m}^{\mathcal{P}}\left(\widehat{v}_{(m, \gamma)}\right)$ as in (19) (green diamonds). Threshold $\alpha=0.05$ is displayed by a dashed horizontal line.

Using Corollary 6.3, one can build a modified version of the empirical accessible test statistic $\phi_{m}^{\mathcal{P}}$ in (9) with asymptotic level $\alpha$, adapted to large thresholds. Under $H_{0}(t)$ hypothesis, let $\theta$ be the median value of the distribution and $u_{(m, \gamma)} \in \mathbb{R}_{+}^{\star}$ so that $\theta+u_{(m, \gamma)}:=F^{-1}\left(1-\frac{1}{m^{\gamma}}\right)$, and $\theta-u_{(m, \gamma)}:=F^{-1}\left(\frac{1}{m^{\gamma}}\right)$. Coming back to the ratio $R_{m, \theta}$ introduced in Section 4 , we have

$$
R_{m, \theta}\left(u_{(m, \gamma)}\right):=\frac{\check{\mathcal{P}}_{m}\left(\theta-u_{(m, \gamma)}\right)}{\check{\mathcal{P}}_{m}\left(\theta+u_{(m, \gamma)}\right)} .
$$

Then, from Corollary 6.3, it holds that

$\widehat{v}_{(m, \gamma)}\left(R_{m, \theta}\left(u_{(m, \gamma)}\right)-1\right) \underset{m \rightarrow \infty}{\stackrel{d, H_{0}}{\longrightarrow}} \mathcal{N}(0,1) \quad$ with $\quad \widehat{v}_{(m, \gamma)}=\sqrt{\frac{m^{2} \check{\mathcal{P}}\left(\theta-u_{(m, \gamma)}\right)}{8}}$.

This asymptotic allows us to define a new symmetry test statistic by prescribing

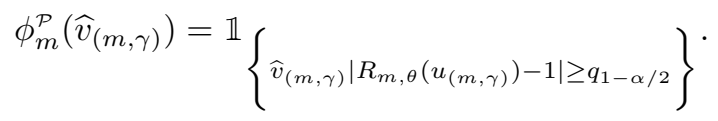

Conversely to Equation (9), the main statistical interest of the test in (19) is to avoid the estimation of the variance. Furthermore, as one can remark for instance in the numerical study in Figure 8, global performances of the test in (19) are similar to those obtained in the same distributional framework in Figure 4. This means that we can obtain similar $p$-values with less effort in terms of moment estimations. Furthermore, notice that, since $\gamma=\frac{-\ln \left(p_{u_{m}, \gamma}\right)}{\ln (m)}$ and $\gamma \in(0,2)$, the range of admissible values for $u_{m, \gamma}$ from Proposition 6.2 is growing with $m$.

In order to better appreciate the statistical equivalent performance of the four proposed test statistics, we gathered in Table 1 averaged values of the empirical 
$\mathbb{P}_{H_{0}(t)}\left(\phi_{m}^{\mathcal{P}}=1\right)$ and associated standard deviations for different thresholds and several distributional models. We underline in bold some values for which the last proposed test in (19) seems slightly outperform the other ones especially for large threshold values (see Table 1).

TABLE 1

Under $H_{0}$ hypothesis. Average of the empirical values of $\mathbb{P}_{H_{0}(t)}\left(\phi_{m}^{\mathcal{P}}=1\right)$ and associated standard deviations for different thresholds $t_{m, \gamma}$, for 500 Montecarlo simulations, for $m=1024$. We consider different test statistics: $\phi_{m}^{\mathcal{P}}\left(\widehat{\sigma}^{\mathcal{P}}\right)$ as in $(12), \phi_{m}^{\mathcal{P}}\left(\widehat{\sigma}^{\mathcal{A}}\right)$ as in $(13)$, $\phi_{m}^{k-s}\left(\widehat{\sigma}^{k-s}\right)$ as in (15) and finally $\phi_{m}^{\mathcal{P}}\left(\widehat{v}_{(m, \gamma)}\right)$ as in $(19)$.

Gaussian marginal distribution $\mathcal{N}(0,1)$

\begin{tabular}{l|c|c|c|c} 
Thresholds $t_{m, \gamma}$ & 1.0 & 1.85 & 2.69 & 3.54 \\
\hline $\mathbb{P}_{H_{0}}\left(\phi_{m}^{\mathcal{P}}\left(\widehat{\sigma}^{\mathcal{P}}\right)=1\right)$ & $0.0(0.0)$ & $0.018(0.133)$ & $0.048(0.214)$ & $0.040(0.196)$ \\
$\mathbb{P}_{H_{0}}\left(\phi_{m}^{\mathcal{A}}\left(\widehat{\sigma}^{\mathcal{A}}\right)=1\right)$ & $0.0(0.0)$ & $0.018(0.133)$ & $0.048(0.214)$ & $0.038(0.191)$ \\
$\mathbb{P}_{H_{0}}\left(\phi_{m}^{\mathrm{k}-\mathrm{s}}\left(\widehat{\sigma}^{\mathrm{k}-\mathrm{s}}\right)=1\right)$ & $0.0(0.0)$ & $0.018(0.133)$ & $0.050(0.218)$ & $0.040(0.196)$ \\
$\mathbb{P}_{H_{0}}\left(\phi_{m}^{\mathcal{P}}(\widehat{v})=1\right)$ & $0.0(0.0)$ & $\mathbf{0 . 0 1 6}(\mathbf{0 . 1 2 5})$ & $0.048(0.214)$ & $0.038(0.191)$ \\
& Student marginal distribution $d f=3$
\end{tabular}

\begin{tabular}{l|c|c|c|c} 
Thresholds $t_{m, \gamma}$ & 1.5 & 3.62 & 5.75 & 7.87 \\
\hline $\mathbb{P}_{H_{0}}\left(\phi_{m}^{\mathcal{P}}\left(\widehat{\sigma}^{\mathcal{P}}\right)=1\right)$ & $0.006(0.077)$ & $0.01(0.099)$ & $0.034(0.181)$ & $0.038(0.191)$ \\
$\mathbb{P}_{H_{0}}\left(\phi_{m}^{\mathcal{A}}\left(\widehat{\sigma}^{\mathcal{A}}\right)=1\right)$ & $0.006(0.077)$ & $0.01(0.099)$ & $0.034(0.181)$ & $0.038(0.191)$ \\
$\mathbb{P}_{H_{0}}\left(\phi_{m}^{\mathrm{k}-\mathrm{s}}\left(\widehat{\sigma}^{\mathrm{k}-\mathrm{s}}\right)=1\right)$ & $0.002(0.045)$ & $0.01(0.099)$ & $0.032(0.176)$ & $0.038(0.191)$ \\
$\mathbb{P}_{H_{0}}\left(\phi_{m}^{\mathcal{P}}(\widehat{v})=1\right)$ & $\mathbf{0 . 0} \mathbf{( 0 . 0 )}$ & $0.01(0.099)$ & $0.032(0.176)$ & $0.038(0.191)$ \\
& Uniform marginal distribution $\mathcal{U}(0,1)$
\end{tabular}

\begin{tabular}{l|c|c|c|c} 
Thresholds $t_{m, \gamma}$ & 0.7 & 0.78 & 0.85 & 0.93 \\
\hline $\mathbb{P}_{H_{0}}\left(\phi_{m}^{\mathcal{P}}\left(\widehat{\sigma}^{\mathcal{P}}\right)=1\right)$ & $0.002(0.045)$ & $0.0(0.0)$ & $0.004(0.063)$ & $0.094(0.292)$ \\
$\mathbb{P}_{H_{0}}\left(\phi_{m}^{\mathcal{A}}\left(\widehat{\sigma}^{\mathcal{A}}\right)=1\right)$ & $0.002(0.045)$ & $0.0(0.0)$ & $0.004(0.063)$ & $0.094(0.292)$ \\
$\mathbb{P}_{H_{0}}\left(\phi_{m}^{\mathrm{k}-\mathrm{s}}\left(\widehat{\sigma}^{\mathrm{k}-\mathrm{s}}\right)=1\right)$ & $0.0(0.0)$ & $0.0(0.0)$ & $0.004(0.063)$ & $0.096(0.295)$ \\
$\mathbb{P}_{H_{0}}\left(\phi_{m}^{\mathcal{P}}(\widehat{v})=1\right)$ & $0.0(0.0)$ & $0.0(0.0)$ & $0.0(0.0)$ & $\mathbf{0 . 0 6 4}(\mathbf{0 . 2 4 5})$
\end{tabular}

\section{Proofs}

Proof of Proposition 3.1. One can start by observing that both $f_{1}^{(t)}(l, k)$ and $f_{2}^{(t)}(k, l)$ follow a Bernoulli distribution with parameter $2 p_{t}\left(1-p_{t}\right)$. Thus, using Equation (1), one can write

$$
\begin{aligned}
\mathbb{E}\left(\mathcal{P}_{m}(t)\right) & =\mathbb{E}\left(\mathcal{P}_{m}^{(1)}(t)+\mathcal{P}_{m}^{(2)}(t)\right)=2 \sum_{l=1}^{m} \sum_{k=2}^{m} \mathbb{E}\left(f_{1}^{(t)}(l, k)\right) \\
& =4 m(m-1) p_{t}\left(1-p_{t}\right) .
\end{aligned}
$$

Thus we obtain the result for $\mathbb{E}\left(\check{\mathcal{P}}_{m}\right)$ dividing by $m^{2}$.

Proof of Proposition 3.2. Without loss of generality, one can assume that $t \leq s$. We use here Definition (1) of the perimeter. Let us start by making use of the fact that $\left(\mathcal{P}_{m}^{(1)}(s), \mathcal{P}_{m}^{(1)}(t)\right) \stackrel{d}{=}\left(\mathcal{P}_{m}^{(2)}(s), \mathcal{P}_{m}^{(2)}(t)\right)$. Thus,

$$
\operatorname{Cov}\left(\mathcal{P}_{m}(s), \mathcal{P}_{m}(t)\right)=2 \operatorname{Cov}\left(\mathcal{P}_{m}^{(1)}(s), \mathcal{P}_{m}^{(1)}(t)\right)+2 \operatorname{Cov}\left(\mathcal{P}_{m}^{(2)}(s), \mathcal{P}_{m}^{(1)}(t)\right)
$$


Firstly,

$$
\begin{aligned}
\operatorname{Cov}\left(\mathcal{P}_{m}^{(1)}(s), \mathcal{P}_{m}^{(1)}(t)\right) & =\operatorname{Cov}\left(\sum_{l=1}^{m} \sum_{k=2}^{m} f_{1}^{(s)}(l, k), \sum_{i=1}^{m} \sum_{j=2}^{m} f_{1}^{(t)}(i, j)\right) \\
& =\sum_{l=1}^{m} \sum_{k=2}^{m} \sum_{j=2}^{m} \operatorname{Cov}\left(f_{1}^{(s)}(l, k), f_{1}^{(t)}(i, j)\right),
\end{aligned}
$$

where we have used that for $\forall k, l$, if $, i \neq l, \operatorname{Cov}\left(f_{1}^{(s)}(l, k), f_{1}^{(t)}(i, j)\right)=0$. In the above sum, there will be only three cases for which the covariance function is different from $0: j=k, j=k+1$ and $j=k-1$.

1. If $j=k$,

$$
\begin{aligned}
& \mathbb{E}\left(f_{1}^{(s)}(l, k) f_{1}^{(t)}(l, k)\right)=\mathbb{P}\left(f_{1}^{(s)}(l, k)=1 \cap f_{1}^{(t)}(l, k)=1\right) \\
&=\mathbb{P}\left(\left(\left(X_{l, k-1}<s \cap X_{l, k} \geq s\right) \cup\left(X_{l, k-1} \geq s \cap X_{l, k}<s\right)\right)\right. \\
&\left.\cap\left(\left(X_{l, k-1}<t \cap X_{l, k} \geq t\right) \cup\left(X_{l, k-1} \geq t \cap X_{l, k}<t\right)\right)\right) \\
&= \mathbb{P}\left(\left(X_{l, k-1}<s \cap X_{l, k} \geq s \cap X_{l, k-1}<t \cap X_{l, k} \geq t\right)\right. \\
& \cup(X_{l, k-1}<s \cap \underbrace{X_{l, k} \geq s \cap X_{l, k}<t}_{=\emptyset} \cap X_{l, k-1} \geq t) \\
& \cup(\underbrace{X_{l, k-1} \geq s \cap X_{l, k-1}<t}_{=\emptyset} \cap X_{l, k}<s \cap X_{l, k} \geq t) \\
&\left.\cup\left(X_{l, k-1} \geq s \cap X_{l, k}<s \cap X_{l, k-1} \geq t \cap X_{l, k}<t\right)\right) \\
&= \mathbb{P}\left(X_{l, k-1}<t \cap X_{l, k} \geq s\right)+\mathbb{P}\left(X_{l, k-1} \geq s \cap X_{l, k}<t\right) \\
&= p_{s}\left(1-p_{t}\right)+\left(1-p_{t}\right) p_{s} \\
&= 2 p_{s}\left(1-p_{t}\right) .
\end{aligned}
$$

Thus,

$$
\begin{aligned}
\operatorname{Cov}\left(f_{1}^{(s)}(l, k), f_{1}^{(t)}(l, k)\right) & =2 p_{s}\left(1-p_{t}\right)-2 p_{s}\left(1-p_{s}\right) 2 p_{t}\left(1-p_{t}\right) \\
& =2 p_{s}\left(1-p_{t}\right)\left(1-2 p_{t}+2 p_{t} p_{s}\right) .
\end{aligned}
$$

2. If $j=k+1$,

$$
\begin{aligned}
\mathbb{E}\left(f_{1}^{(s)}(l, k) f_{1}^{(t)}(l, k+1)\right)=\mathbb{P}\left(f_{1}^{(s)}(l, k)=1 \cap f_{1}^{(t)}(l, k+1)=1\right) \\
=\mathbb{P}\left(\left(\left(X_{l, k-1}<s \cap X_{l, k} \geq s\right) \cup\left(X_{l, k-1} \geq s \cap X_{l, k}<s\right)\right)\right. \\
\left.\cap\left(\left(X_{l, k}<t \cap X_{l, k+1} \geq t\right) \cup\left(X_{l, k} \geq t \cap X_{l, k+1}<t\right)\right)\right) \\
=\mathbb{P}\left((X_{l, k-1}<s \cap \underbrace{X_{l, k} \geq s \cap X_{l, k}<t}_{=\emptyset} \cap X_{l, k+1} \geq t)\right.
\end{aligned}
$$




$$
\begin{aligned}
& \cup\left(X_{l, k-1}<s \cap X_{l, k} \geq s \cap X_{l, k} \geq t \cap X_{l, k+1}<t\right) \\
& \cup\left(X_{l, k-1} \geq s \cap X_{l, k}<s \cap X_{l, k}<t \cap X_{l, k+1} \geq t\right) \\
& \left.\cup\left(X_{l, k-1} \geq s \cap X_{l, k}<s \cap X_{l, k} \geq t \cap X_{l, k+1}<t\right)\right) \\
= & \mathbb{P}\left(X_{l, k-1}<s\right) \mathbb{P}\left(X_{l, k} \geq s\right) \mathbb{P}\left(X_{l, k+1}<t\right) \\
& +\mathbb{P}\left(X_{l, k-1} \geq s\right) \mathbb{P}\left(X_{l, k}<t\right) \mathbb{P}\left(X_{l, k+1} \geq t\right) \\
& +\mathbb{P}\left(X_{l, k-1} \geq s\right) \mathbb{P}\left(X_{l, k} \geq t \cap X_{l, k}<s\right) \mathbb{P}\left(X_{l, k+1}<t\right) \\
= & \left(1-p_{s}\right) p_{s}\left(1-p_{t}\right)+\left(1-p_{t}\right) p_{t} p_{s}+p_{s}\left(p_{t}-p_{s}\right)\left(1-p_{t}\right) \\
= & p_{s}\left(1-p_{t}\right)\left(1+2 p_{t}-2 p_{s}\right) .
\end{aligned}
$$

Thus,

$$
\begin{aligned}
\operatorname{Cov}\left(f_{1}^{(s)}(l, k), f_{1}^{(t)}(l, k+1)\right)= & p_{s}\left(1-p_{t}\right)\left(1+2 p_{t}-2 p_{s}\right) \\
& -2 p_{s}\left(1-p_{s}\right) 2 p_{s}\left(1-p_{t}\right) \\
= & p_{s}\left(1-p_{t}\right)\left(1-2 p_{t}-2 p_{s}+4 p_{t} p_{s}\right) .
\end{aligned}
$$

3. Similarly, if $j=k-1$,

$$
\begin{aligned}
\operatorname{Cov}\left(f_{1}^{(s)}(l, k), f_{1}^{(t)}(l, k-1)\right) & =\operatorname{Cov}\left(f_{1}^{(s)}(l, k), f_{1}^{(t)}(l, k+1)\right) \\
& =p_{s}\left(1-p_{t}\right)\left(1-2 p_{t}-2 p_{s}+4 p_{t} p_{s}\right) .
\end{aligned}
$$

Thus,

$$
\begin{aligned}
\operatorname{Cov}\left(\mathcal{P}_{m}^{(1)}(s), \mathcal{P}_{m}^{(1)}(t)\right)= & \sum_{l=1}^{m} \sum_{k=2}^{m} \operatorname{Cov}\left(f_{1}^{(s)}(l, k), f_{1}^{(t)}(l, k)\right) \\
& +\sum_{l=1}^{m} \sum_{k=3}^{m} \operatorname{Cov}\left(f_{1}^{(s)}(l, k), f_{1}^{(t)}(l, k+1)\right) \\
& +\sum_{l=1}^{m} \sum_{k=3}^{m} \operatorname{Cov}\left(f_{1}^{(s)}(l, k), f_{1}^{(t)}(l, k-1)\right) \\
= & 2 m(m-1) p_{s}\left(1-p_{t}\right)\left(1-2 p_{t}\left(1-p_{s}\right)\right) \\
& +2 m(m-2) p_{s}\left(1-p_{t}\right)\left(1+2 p_{t}-2 p_{s}-4 p_{t}\left(1-p_{s}\right)\right) .
\end{aligned}
$$

Now, let us compute the inter-level covariance between the horizontal contributions to the perimeter and the vertical ones:

$$
\begin{aligned}
\operatorname{Cov}\left(\mathcal{P}_{m}^{(1)}(s), \mathcal{P}_{m}^{(2)}(t)\right) & =\operatorname{Cov}\left(\sum_{l=1}^{m} \sum_{k=2}^{m} f_{1}^{(s)}(l, k), \sum_{i=1}^{m} \sum_{j=2}^{m} f_{2}^{(t)}(j, i)\right) \\
& =\sum_{l=1}^{m} \sum_{k=2}^{m} \sum_{i=1}^{m} \sum_{j=2}^{m} \operatorname{Cov}\left(f_{1}^{(s)}(l, k), f_{2}^{(t)}(j, i)\right) .
\end{aligned}
$$

To this aim, notice that the covariance between cells is non equal to zero only if the cells are neighbors, that implies that 

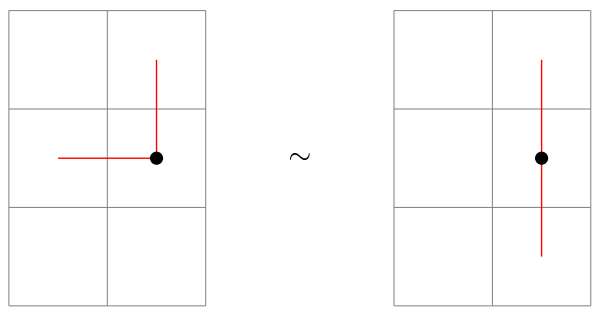

FIG 9. Representation of the configuration between $\left(f_{1}(l, k), f_{2}(l+1, k-1)\right)$ (left panel) and $\left(f_{1}(l, k), f_{1}(l, k+1)\right)$ (right panel), the black dot represents the cell $(k, l)$.

for $i \notin\{k-1, k\}$ or for $l \notin\{j-1, j\} \operatorname{Cov}\left(f_{1}(l, k), f_{2}(j, i)\right)=0$.

Thus,

$$
\begin{aligned}
\operatorname{Cov}\left(\mathcal{P}_{m}^{1}(s), \mathcal{P}_{m}^{2}(t)\right)=\sum_{k=2}^{m} \sum_{j=2}^{m} & \left(\operatorname{Cov}\left(f_{1}^{(s)}(j-1, k), f_{2}^{(t)}(j, k-1)\right)\right. \\
& +\operatorname{Cov}\left(f_{1}^{(s)}(j, k), f_{2}^{(t)}(j, k-1)\right) \\
& +\operatorname{Cov}\left(f_{1}^{(s)}(j-1, k), f_{2}^{(t)}(j-1, k)\right) \\
& \left.+\operatorname{Cov}\left(f_{1}^{(s)}(j, k), f_{2}^{(t)}(j, k)\right)\right) .
\end{aligned}
$$

Due to the i.i.d setting (see Figure 9), one can remark that the four types of covariance in the above sum are equal and are equal to $\operatorname{Cov}\left(f_{1}^{(s)}(l, k), f_{2}^{(t)}(l, k+\right.$ 1)).

Thus,

$$
\begin{aligned}
\operatorname{Cov}\left(\mathcal{P}_{m}^{1}(s), \mathcal{P}_{m}^{2}(t)\right) & =4(m-1)^{2} \operatorname{Cov}\left(f_{1}^{(s)}(l, k), f_{2}^{(t)}(l, k+1)\right) \\
& =4(m-1)^{2} p_{s}\left(1-p_{t}\right)\left(1+2 p_{t}-2 p_{s}-4 p_{t}\left(1-p_{s}\right)\right) .
\end{aligned}
$$

Putting all elements together, we get the covariance function of the unscaled perimeter:

$$
\begin{aligned}
\operatorname{Cov}\left(\mathcal{P}_{m}(s), \mathcal{P}_{m}(t)\right)= & 4 p_{s}\left(1-p_{t}\right)\left(\left(4 m^{2}-7 m+2\right)\right. \\
& -2 p_{t}\left(1-p_{s}\right)\left(7 m^{2}-13 m+4\right) \\
& \left.+2\left(p_{t}-p_{s}\right)\left(3 m^{2}-6 m+2\right)\right) .
\end{aligned}
$$

Hence, the result.

Proof of Theorem 3.3. In order to prove this result, we will use the well known Cramèr-Wold method. For the sake of completeness we recall it below.

Theorem 7.1 (Cramèr-Wold method, see, e.g., [6], p. 383). Let $\bar{X}^{m}=\left(X_{1}^{m}\right.$, $\left.\cdots, X_{r}^{m}\right)$ and $\bar{X}=\left(X_{1}, \cdots, X_{r}\right)$ be random vectors of dimension $r \geq 1$. Then, $\bar{X}^{m}$ converges in distribution to $\bar{X}$ if and only if 


$$
\sum_{i=1}^{r} a_{i} X_{i}^{m} \underset{m \rightarrow+\infty}{\stackrel{d}{\longrightarrow}} \sum_{i=1}^{r} a_{i} X_{i}
$$

for each $\left(a_{1}, \cdots, a_{r}\right) \in \mathbb{R}^{r}$, i.e., if every fixed linear combination of the coordinates of $\bar{X}^{m}$ converges in distribution to the correspondent linear combination of coordinates of $\bar{X}$.

We first need to introduce some notations. Let $V_{m}=\{1, \ldots, m\} \times\{2, \ldots, m\}$ $a_{1}, \ldots, a_{r} \in \mathbb{R}, t_{1}, \ldots, t_{r} \in \mathbb{R},(l, k) \in V_{m}$ and $i \in\{1, \ldots, r\}$. Let $\mathcal{P}_{m}\left(t_{i}\right)$ as in Equation (1). We introduce

$$
\begin{aligned}
Y_{l, k}\left(t_{i}\right)= & \mathbb{1}_{\left(Z_{l, k-1}^{\left(t_{i}\right)}=0 \cap Z_{l, k}^{\left(t_{i}\right)}=1\right) \cup\left(Z_{l, k-1}^{\left(t_{i}\right)}=1 \cap Z_{l, k}^{\left(t_{i}\right)}=0\right)} \\
& +\mathbb{1}_{\left(Z_{k-1, l}^{\left(t_{i}\right)}=0 \cap Z_{k, l}^{\left(t_{i}\right)}=1\right) \cup\left(Z_{k-1, l}^{\left(t_{i}\right)}=1 \cap Z_{k, l}^{\left(t_{i}\right)}=0\right)} .
\end{aligned}
$$

Note that $0 \leq Y_{l, k}\left(t_{i}\right) \leq 2$ a.s. and $\left|Y_{l, k}\left(t_{i}\right)-\mathbb{E}\left(Y_{l, k}\left(t_{i}\right)\right)\right| \leq 2$, thus $\left|\sum_{i=1}^{r} a_{i}\left(Y_{k, l}\left(t_{i}\right)-\mathbb{E}\left(Y_{l, k}\left(t_{i}\right)\right)\right)\right| \leq 2 \sum_{i=1}^{r}\left|a_{i}\right|$. We note

$$
\left.W_{l, k}^{m}=\frac{1}{m} \sum_{i=1}^{r} a_{i}\left[Y_{l, k}\left(t_{i}\right)-\mathbb{E}\left(Y_{l, k}\left(t_{i}\right)\right)\right)\right],
$$

and finally,

$S_{m}=\sum_{l=1}^{m} \sum_{k=2}^{m} W_{l, k}^{m}=\frac{a_{1}}{m}\left(\mathcal{P}_{m}\left(t_{1}\right)-\mathbb{E}\left(\mathcal{P}_{m}\left(t_{1}\right)\right)\right)+\ldots+\frac{a_{r}}{m}\left(\mathcal{P}_{m}\left(t_{r}\right)-\mathbb{E}\left(\mathcal{P}_{m}\left(t_{r}\right)\right)\right)$.

Using the Cramèr-Wold method, we need to prove that

$$
S_{m} \underset{m \rightarrow \infty}{\stackrel{d}{\longrightarrow}} \mathcal{N}\left(0, \sum_{i=1}^{r} a_{i}^{2} \Sigma_{r}^{\star}(i, i)+\sum_{0 \leq i<j \leq r} 2 a_{i} a_{j} \Sigma_{r}^{\star}(i, j)\right),
$$

with $\Sigma_{r}^{\star}$ as in (5). To this purpose we use the following weaker version of Theorem 1 of [20] for bounded variables (see p.200 in [20] for details).

Proposition 7.2. Let $V_{m}=\{1, \ldots, m\} \times\{2, \ldots, m\}$ and $\left\{X_{z}^{m}, z \in V_{m}\right\}$ be a sequence of 1-dependent random field, and $S_{m}=\sum_{z \in V_{m}} X_{z}^{m}$. If the following three conditions are satisfied:

(i) $\forall \epsilon>0, \sum_{z \in V_{m}} \mathbb{P}\left(\left|X_{z}^{m}\right| \geq \epsilon\right) \underset{m \rightarrow \infty}{\longrightarrow} 0$,

(ii) $\sum_{z \in V_{m}} \mathbb{E}\left(\left(X_{z}^{m}\right)^{2}\right) \leq C<+\infty$,

(iii) $\mathbb{E}\left(S_{m}\right) \underset{m \rightarrow \infty}{\longrightarrow} 0$ and $\operatorname{Var}\left(S_{m}\right) \underset{m \rightarrow \infty}{\longrightarrow} \sigma^{2}$ with $\sigma^{2} \in(0, \infty)$,

then, $S_{m} \underset{m \rightarrow \infty}{\stackrel{d}{\longrightarrow}} \mathcal{N}\left(0, \sigma^{2}\right)$.

We start by showing that $\left\{W_{z}^{m}, z \in V_{m}\right\}$ satisfies (i). For $\epsilon>0$, we get that,

$$
\sum_{(l, k) \in V_{m}} \mathbb{P}\left(\left|W_{l, k}^{m}\right| \geq \epsilon\right) \leq \sum_{(l, k) \in V_{m}} \frac{\mathbb{E}\left(\left|W_{l, k}^{m}\right|^{3}\right)}{\epsilon^{3}}
$$




$$
\leq \frac{\left(2 \sum_{i=1}^{r}\left|a_{i}\right|\right)^{3} \times m(m-1)}{(m \epsilon)^{3}} \underset{m \rightarrow \infty}{\longrightarrow} 0 .
$$

To prove that $\left\{W_{z}^{m}, z \in V_{m}\right\}$ satisfies (ii), we write

$$
\sum_{(l, k) \in V_{m}} \mathbb{E}\left(\left(W_{l, k}^{m}\right)^{2}\right) \leq m(m-1)\left(\frac{2}{m} \sum_{i=1}^{r}\left|a_{i}\right|\right)^{2} \leq\left(2 \sum_{i=1}^{r}\left|a_{i}\right|\right)^{2} .
$$

For (iii) we observe that $\operatorname{Var}\left(S_{m}\right)=\operatorname{Var}\left(\frac{a_{1}}{m} \mathcal{P}_{m}\left(t_{1}\right)+\ldots+\frac{a_{r}}{m} \mathcal{P}_{m}\left(t_{r}\right)\right)$ thus, as $m \rightarrow \infty$,

$\operatorname{Var}\left(\frac{a_{1}}{m} \mathcal{P}_{m}\left(t_{1}\right)+\ldots+\frac{a_{r}}{m} \mathcal{P}_{m}\left(t_{r}\right)\right) \rightarrow \sum_{i=1}^{r} a_{i}^{2} \Sigma_{r}^{\star}(i, i)+\sum_{0 \leq i<j \leq r} 2 a_{i} a_{j} \Sigma_{r}^{\star}(i, j)$, by Equation (5). Using the auxiliary Proposition 7.2 proves Theorem 3.3.

Proof of Proposition 3.5. Let $\theta$ be the median value of the underlying distribution. We first start with solving the quadratic Equation (6) for $m=2$ under the condition that $t>\theta\left(\right.$ i.e $\left.p(t)<\frac{1}{2}\right)$. We get that $p(t)=\frac{1}{2}\left(1-\sqrt{1-2 \mathbb{E}\left(\check{\mathcal{P}}_{2}(t)\right)}\right)$. Since we are in an i.i.d. framework, the variables $\check{\mathcal{P}}_{2}^{i}(t)$ for each sub-image $S_{i}$ are i.i.d, and, by using the Law of Large numbers, $\bar{S}_{m}(t):=\frac{4}{m^{2}} \sum_{i} \check{\mathcal{P}}_{2}^{i}(t)$ is an estimator of $\mathbb{E}\left(\check{\mathcal{P}}_{2}(t)\right)$. Applying the Central Limit Theorem, we get

$$
\sqrt{\frac{m^{2}}{4}}\left(\bar{S}_{m}(t)-\mathbb{E}\left(\check{\mathcal{P}}_{2}(t)\right)\right) \underset{m \rightarrow \infty}{\stackrel{d}{m}}, \mathcal{N}\left(0, \sigma_{\check{\mathcal{P}}}^{2}(t, 2)\right),
$$

with $\sigma_{\breve{\mathcal{P}}}^{2}(t, 2)=\operatorname{Var}\left(\check{\mathcal{P}}_{2}(t)\right)=p_{t}\left(1-p_{t}\right)\left(1-3 p_{t}\left(1-p_{t}\right)\right)$. We apply the Delta method to Equation (21) using the function $g$ as defined in Proposition 3.4. Note that $g$ is differentiable on $\left(0, \frac{1}{2}\right)$ and that for $t \neq \frac{1}{2}, \mathbb{E}\left(\check{\mathcal{P}}_{2}(t)\right)<\frac{1}{2}$ Thus

$$
\sqrt{\frac{m^{2}}{4}}\left(g\left(\bar{S}_{m}(t)\right)-g\left(\mathbb{E}\left(\check{\mathcal{P}}_{2}(t)\right)\right)\right) \underset{m \rightarrow \infty}{\stackrel{d}{\longrightarrow}} g^{\prime}\left(\mathbb{E}\left(\check{\mathcal{P}}_{2}(t)\right) \mathcal{N}\left(0, \sigma_{\tilde{\mathcal{P}}}^{2}(t, 2)\right),\right.
$$

with $g^{\prime}(x)=\frac{1}{2 \sqrt{1-2 x}}$. Since $\sqrt{1-2 \mathbb{E}\left(\check{\mathcal{P}}_{2}(t)\right)}=1-2 p_{t}$, we get

$$
m\left(\widehat{p}_{m}^{\mathcal{P}}(t)-p(t)\right) \underset{m \rightarrow \infty}{\stackrel{d}{\longrightarrow}} \mathcal{N}\left(0, \frac{p(t)(1-p(t))(1-3 p(t)(1-p(t)))}{4(1-2 p(t))^{2}}\right) .
$$

Hence, the result for $\widehat{p}_{m}^{\mathcal{P}}$. For $\widehat{p}_{m}^{\mathcal{A}}$, the result is a classical consequence of the Central Limit Theorem.

Proof of Proposition 4.1. Let $t, s \in \mathbb{R}$ with $t<s$. Let

$$
\mu:=\lim _{m \rightarrow \infty}\left(\begin{array}{c}
\mu_{\check{\mathcal{P}}_{m}}(t) \\
\mu_{\check{\mathcal{P}}_{m}}(s)
\end{array}\right)=\left(\begin{array}{c}
4 p_{t}\left(1-p_{t}\right) \\
4 p_{s}\left(1-p_{s}\right)
\end{array}\right) \text { and } g:\left(\begin{array}{l}
x \\
y
\end{array}\right) \mapsto \frac{x}{y} .
$$


Applying the Delta method to the multivariate Central Limit Theorem 3.3, we get the following result

$$
\begin{gathered}
m\left(\frac{\check{\mathcal{P}}_{m}(t)}{\check{\mathcal{P}}_{m}(s)}-\frac{\mu_{\check{\mathcal{P}}_{m}}(t)}{\mu_{\check{\mathcal{P}}_{m}}(s)}\right) \underset{m \rightarrow \infty}{\stackrel{d}{\longrightarrow}} \mathcal{N}\left(0, \nabla g(\mu)^{T} \Sigma_{2}^{\star} \nabla g(\mu)\right), \\
\text { with } \nabla g(\mu)=\left(\begin{array}{c}
\frac{1}{4 p_{s}\left(1-p_{s}\right)} \\
\left.\frac{-4 p_{t}\left(1-p_{t}\right)}{\left(4 p_{s}\left(1-p_{s}\right)\right)^{2}}\right)
\end{array}\right) \text { and } \Sigma_{2}^{\star} \text { as in Theorem 3.3. Thus, } \\
\begin{array}{c}
\nabla g(\mu)^{T} \Sigma_{2}^{\star} \nabla g(\mu)=\frac{p_{t}\left(1-p_{t}\right)}{\left(p_{s}\left(1-p_{s}\right)\right)^{3}}\left(p_{s}\left(1-p_{s}\right)-2 p_{s}\left(1-p_{t}\right)-3 p_{s}\left(p_{t}-p_{s}\right)\left(1-p_{t}\right)\right. \\
\left.+p_{t}\left(1-p_{t}\right)\right) .
\end{array}
\end{gathered}
$$

Proof of Proposition 4.2. From Proposition 4.1 it holds that,

$$
m\left(R_{m, \theta}(t)-\frac{\mu_{\check{\mathcal{P}}_{m}}(\theta-t)}{\mu_{\check{\mathcal{P}}_{m}}(\theta+t)}\right) \underset{m \rightarrow \infty}{\stackrel{d, H_{0}}{\longrightarrow}} \mathcal{N}\left(0, \widetilde{\sigma}^{2}(\theta-t, \theta+t)\right) .
$$

Let us denote $\sigma:=\sigma(\theta+t)=\sqrt{\frac{\left(2 p_{\theta+t}-1\right)\left(3 p_{\theta+t}-2\right)}{p_{\theta+t}\left(1-p_{\theta+t}\right)^{2}}}$ the standard deviation of the ratio under $H_{0}(t)$ and $\widetilde{\sigma}$ the standard deviation in $(22)$. Let $\phi_{m}^{\mathcal{P}}(\sigma)$ as in Equation (8).

Under the alternative hypothesis $H_{1}(t), p_{\theta-t} \neq 1-p_{\theta+t}$. Besides, $p_{\theta-t} \neq p_{\theta+t}$. Let define $\delta=1-\frac{p_{\theta-t}\left(1-p_{\theta-t}\right)}{p_{\theta+t}\left(1-p_{\theta+t}\right)}$. By hypothesis $\delta \neq 0$. Recall that

$$
\frac{\mu_{\check{\mathcal{P}}_{m}}(\theta-t)}{\mu_{\check{\mathcal{P}}_{m}}(\theta+t)}=\frac{p_{\theta-t}\left(1-p_{\theta-t}\right)}{p_{\theta+t}\left(1-p_{\theta+t}\right)}, \quad \text { then } \quad \frac{\mu_{\check{\mathcal{P}}_{m}}(\theta-t)}{\mu_{\check{\mathcal{P}}_{m}}(\theta+t)}=1-\delta
$$

Then, we get

$$
\begin{aligned}
\mathbb{P}_{H_{1}(t)}\left(\phi_{m}^{\mathcal{P}}(\sigma)=1\right)= & \mathbb{P}_{H_{1}(t)}\left(\left|\frac{m}{\widetilde{\sigma}}\left(R_{m, \theta}(t)-1+\delta-\delta\right)\right| \geq q_{1-\alpha / 2} \frac{\sigma}{\widetilde{\sigma}}\right) \\
= & \mathbb{P}_{H_{1}(t)}\left(\frac{m}{\widetilde{\sigma}}\left(R_{m, \theta}(t)-\frac{\mu_{\check{\mathcal{P}}_{m}}(\theta-t)}{\mu_{\check{\mathcal{P}}_{m}}(\theta+t)}\right)-\frac{m}{\widetilde{\sigma}} \delta \geq q_{1-\alpha / 2} \frac{\sigma}{\widetilde{\sigma}}\right) \\
& +\mathbb{P}_{H_{1}(t)}\left(\frac{m}{\widetilde{\sigma}}\left(R_{m, \theta}(t)-\frac{\mu_{\check{\mathcal{P}}_{m}}(\theta-t)}{\mu_{\check{\mathcal{P}}_{m}}(\theta+t)}\right)-\frac{m}{\widetilde{\sigma}} \delta \leq-q_{1-\alpha / 2} \frac{\sigma}{\widetilde{\sigma}}\right) .
\end{aligned}
$$

Then

$$
\mathbb{P}_{H_{1}(t)}\left(\phi_{m}^{\mathcal{P}}(\sigma) \underset{m \rightarrow \infty}{\longrightarrow} 1,\right.
$$

hence, we get the result. 
Proof of Corollary 4.3. Considering the continuous mapping $\psi: x \mapsto\left(\frac{(2 x-1)(3 x-2)}{x(1-x)^{2}}\right)^{-\frac{1}{2}}$ and the fact that $\widehat{p}_{m}(\theta+t)$ is a robust estimator of $p(\theta+t)$, we get that $\psi\left(\widehat{p}_{m}(\theta+t)\right) \underset{m \rightarrow \infty}{\stackrel{a . s .}{\longrightarrow}} \psi(p(\theta+t))$ where $\psi(p(\theta+t))=\frac{1}{\sigma(\theta+t)}$. Thus, applying the Slutsky Theorem to $(7)$, we get

$$
m \psi\left(\widehat{p}_{m}(\theta+t)\right)\left(R_{m, \theta}(t)-1\right) \underset{m \rightarrow \infty}{\stackrel{d, H_{0}}{\longrightarrow}} \psi(p(\theta+t)) \mathcal{N}\left(0, \sigma^{2}(\theta+t)\right)
$$

Hence the result.

Proof of Proposition 4.5. For the sake of simplicity, let us note $\theta-t=u$ and $\theta+t=s$, for $t \in(0, \infty)$ and $\mu=\left(\begin{array}{c}1-p_{u} \\ 1-p_{s}\end{array}\right)$. We recall that, $\forall x \in \mathbb{R}$

$$
\widehat{F}_{m}\left(x^{-}\right)=\frac{1}{m^{2}} \sum_{i=1}^{m} \sum_{j=1}^{m} \mathbb{1}_{\left\{X_{i, j}<x\right\}}
$$

Furthermore, from the bivariate Central Limit Theorem,

$$
m\left(\left(\begin{array}{c}
\widehat{F}_{m}\left(u^{-}\right) \\
\widehat{F}_{m}\left(s^{-}\right)
\end{array}\right)-\left(\begin{array}{c}
F\left(u^{-}\right) \\
F\left(s^{-}\right)
\end{array}\right)\right) \underset{m \rightarrow \infty}{\stackrel{d}{\longrightarrow}} \mathcal{N}\left(\left(\begin{array}{c}
0 \\
0
\end{array}\right),\left(\begin{array}{cc}
\left(1-p_{u}\right) p_{u} & \left(1-p_{u}\right) p_{s} \\
\left(1-p_{u}\right) p_{s} & \left(1-p_{s}\right) p_{s}
\end{array}\right)\right)
$$

Applying the Delta method using the function $g:\left(\begin{array}{l}x \\ y\end{array}\right) \mapsto \frac{x}{1-y}$, it holds that

$$
m\left(\frac{\widehat{F}_{m}\left(u^{-}\right)}{1-\widehat{F}_{m}\left(s^{-}\right)}-1\right) \underset{m \rightarrow \infty}{\stackrel{d}{\longrightarrow}} \mathcal{N}\left(0, \nabla g(\mu)^{T}\left(\begin{array}{cc}
\left(1-p_{u}\right) p_{u} & \left(1-p_{u}\right) p_{s} \\
\left(1-p_{u}\right) p_{s} & \left(1-p_{s}\right) p_{s}
\end{array}\right) \nabla g(\mu)\right)
$$

with $\nabla g(\mu)=\left(\begin{array}{c}\frac{1}{p_{s}} \\ \frac{1-p_{u}}{\left(p_{s}\right)^{2}}\end{array}\right)$. Since under $H_{0}(t), p_{u}=1-p_{s}$, then,

$$
\nabla g(\mu)^{T}\left(\begin{array}{cc}
\left(1-p_{u}\right) p_{u} & \left(1-p_{u}\right) p_{s} \\
\left(1-p_{u}\right) p_{s} & \left(1-p_{s}\right) p_{s}
\end{array}\right) \nabla g(\mu)=\left(\frac{1}{p_{s}} \frac{1}{p_{s}}\right)\left(\begin{array}{cc}
p_{u} p_{s} & p_{s}^{2} \\
p_{s}^{2} & p_{u} p_{s}
\end{array}\right)\left(\begin{array}{l}
\frac{1}{p_{s}} \\
\frac{1}{p_{s}}
\end{array}\right)=\frac{2}{p_{s}}
$$

Then,

$$
m\left(\frac{\widehat{F}_{m}\left(u^{-}\right)}{1-\widehat{F}_{m}\left(s^{-}\right)}-1\right) \underset{m \rightarrow \infty}{\stackrel{d, H_{0}}{\longrightarrow}} \mathcal{N}\left(0, \frac{2}{p_{s}}\right) .
$$

Hence the result.

Proof of Lemma 6.1. We recall from Equation (3) that we have, $\mu_{\mathcal{P}}\left(p_{t}, m\right)=4 p_{t}\left(1-p_{t}\right) m(m-1)$, and from Equation $(4)$

$$
\sigma_{\mathcal{P}}^{2}\left(p_{t}, m\right)=4 p_{t}\left(1-p_{t}\right)\left(\left(4 m^{2}-7 m+2\right)-2 p_{t}\left(1-p_{t}\right)\left(7 m^{2}-13 m+4\right)\right) .
$$


Then, the ratio is equal to

$$
\frac{\sigma_{\mathcal{P}}^{2}\left(p_{t}, m\right)}{\mu_{\mathcal{P}}\left(p_{t}, m\right)}=\frac{\left(4-\frac{7}{m}+\frac{2}{m^{2}}\right)-2 p_{t}\left(1-p_{t}\right)\left(7-\frac{13}{m}+\frac{4}{m^{2}}\right)}{\left(1-\frac{1}{m}\right)} .
$$

Let $r_{m}^{\star}:=\frac{\left(4-\frac{7}{m}+\frac{2}{m^{2}}\right)}{1-\frac{1}{m}}$, then,

$$
\left|\frac{\sigma_{\mathcal{P}}^{2}\left(p_{t}, m\right)}{\mu_{\mathcal{P}}\left(p_{t}, m\right)}-r_{m}^{\star}\right| \leq\left|\frac{2 p_{t}\left(1-p_{t}\right)\left(7 m-13+\frac{4}{m}\right)}{(m-1)}\right| \leq\left|14 p_{t}\left(1-p_{t}\right)\right| .
$$

Hence, uniformly in $m$,

$$
\frac{\sigma_{\mathcal{P}}^{2}\left(p_{t}, m\right)}{\mu_{\mathcal{P}}\left(p_{t}, m\right)} \underset{p_{t} \rightarrow 0}{\longrightarrow} r_{m}^{\star}
$$

Thus, as $m \rightarrow \infty, \frac{\sigma_{\mathcal{P}}^{2}\left(p_{t}, m\right)}{\mu_{\mathcal{P}}\left(p_{t}, m\right)}$ tends to $4=\lim _{m \rightarrow \infty} r_{m}^{\star}$.

For the second item, we can note that $p_{t}\left(1-p_{t}\right)$ reaches its maximal value at $p_{t}=1 / 2$. It follows that

$$
\frac{\sigma_{\mathcal{P}}^{2}\left(p_{t}, m\right)}{\mu_{\mathcal{P}}\left(p_{t}, m\right)} \geq \frac{\left(4 m^{2}-7 m+2\right)-\frac{1}{2}\left(7 m^{2}-13 m+4\right)}{m(m-1)}=\frac{1}{2} .
$$

Hence, we have proven that for $m$ large enough,

$$
\frac{1}{2} \leq \frac{\sigma_{\mathcal{P}}^{2}\left(p_{t}, m\right)}{\mu_{\mathcal{P}}\left(p_{t}, m\right)} \leq 4,
$$

where the second part of the inequality is due to (23) and (24).

Proof of Proposition 6.2. Let us first notice that $\mathbb{E}\left(\mathcal{P}\left(t_{m, \gamma}\right)\right)=\mathbb{E}\left(\mathcal{P}\left(s_{m, \gamma}\right)\right)$, since $p_{t_{m, \gamma}}=1-\frac{1}{m^{\gamma}}=1-p_{s_{m, \gamma}}$. In order to prove the result, we will establish that $\forall a_{1}, a_{2} \in \mathbb{R}$

$$
\begin{array}{r}
\sqrt{\frac{1}{4 \mathbb{E}\left(\mathcal{P}\left(t_{m, \gamma}\right)\right)}}\left(a_{1}\left(\mathcal{P}\left(t_{m, \gamma}\right)-\mathbb{E}\left(\mathcal{P}\left(t_{m, \gamma}\right)\right)\right)+a_{2}\left(\mathcal{P}\left(s_{m, \gamma}\right)-\mathbb{E}\left(\mathcal{P}\left(s_{m, \gamma}\right)\right)\right)\right) \\
\underset{m \rightarrow \infty}{\stackrel{d}{\longrightarrow}} \mathcal{N}\left(0, a_{1}^{2}+a_{2}^{2}\right) .
\end{array}
$$

To prove this statement, we will follow the same idea as in the proof of Theorem 3.3 , introducing the following notations, similar to (20),

$$
\widetilde{W}_{l, k}^{m}=\sqrt{\frac{1}{4 \mathbb{E}\left(\mathcal{P}\left(t_{m, \gamma}\right)\right)}}\left(a_{1}\left(\mathcal{P}_{m}\left(t_{m, \gamma}\right)-\mathbb{E}\left(\mathcal{P}_{m}\left(t_{m, \gamma}\right)\right)\right)+a_{2}\left(\mathcal{P}_{m}\left(s_{m, \gamma}\right)-\mathbb{E}\left(\mathcal{P}_{m}\left(s_{m, \gamma}\right)\right)\right)\right)
$$

and finally, $S_{m}=\sum_{l=1}^{m} \sum_{k=2}^{m} \widetilde{W}_{l, k}^{m}$.

In order to show that $S_{m} \underset{m \rightarrow \infty}{\stackrel{d}{\longrightarrow}} \mathcal{N}\left(0, a_{1}^{2}+a_{2}^{2}\right)$, we will check that the family 
$\left\{\widetilde{W}_{z}^{m}, z \in V_{m}\right\}$ satisfies the three conditions of Proposition 7.2.

To show that $\left\{\widetilde{W}_{z}^{m}, z \in V_{m}\right\}$ satisfies (i), we proceed as in the proof of Theorem 3.3. For $\epsilon>0$ and choosing $p>\frac{4}{2-\gamma}$, we get that,

$$
\begin{aligned}
\sum_{(l, k) \in V_{m}} \mathbb{P}\left(\left|\widetilde{W}_{l, k}^{m}\right| \geq \epsilon\right) & \leq \sum_{(l, k) \in V_{m}} \frac{\mathbb{E}\left(\left|\widetilde{W}_{l, k}^{m}\right|^{p}\right)}{\epsilon^{p}} \leq \frac{\left(2 \sum_{i=1}^{2}\left|a_{i}\right|\right)^{p} m(m-1)}{\epsilon^{p} \sqrt{\left(4 \times 4 m(m-1) \times \frac{1}{m^{\gamma}}\left(1-\frac{1}{m^{\gamma}}\right)\right)^{p}}}, \\
& \leq C m^{2-\frac{1}{2} p(2-\gamma)},
\end{aligned}
$$

for some positive constant $C$. As $p>\frac{4}{2-\gamma}, C m^{2-\frac{1}{2} p(2-\gamma)} \underset{m \rightarrow \infty}{\longrightarrow} 0$.

To show that $\left\{\widetilde{W}_{z}^{m}, z \in V_{m}\right\}$ satisfies (ii),

$$
\begin{aligned}
\sum_{(l, k) \in V_{m}} \mathbb{E}\left(\left(W_{l, k}^{m}\right)^{2}\right) & =\sum_{(l, k) \in V_{m}} \frac{1}{4 \mathbb{E}\left(\mathcal{P}\left(t_{m, \gamma}\right)\right)} \operatorname{Var}\left(a_{1} Y_{l, k}\left(t_{m, \gamma}\right)+a_{2} Y_{l, k}\left(s_{m, \gamma}\right)\right) \\
& \leq \frac{2 m(m-1)}{4 \mathbb{E}\left(\mathcal{P}\left(t_{m, \gamma}\right)\right)}\left(a_{1}^{2} \operatorname{Var}\left(Y_{1,2}\left(t_{m, \gamma}\right)\right)+a_{2}^{2} \operatorname{Var}\left(Y_{1,2}\left(s_{m, \gamma}\right)\right)\right) .
\end{aligned}
$$

Let us recall that $Y_{1,2}(t)$ is a sum of two Bernoulli random variables with parameter $2 p_{t}\left(1-p_{t}\right)$. Hence, $\operatorname{Var}\left(Y_{1,2}\left(t_{m, \gamma}\right)\right) \leq 2 p_{t_{m, \gamma}}\left(1-p_{t_{m, \gamma}}\right)$.

Since $p_{t_{m, \gamma}}=1-p_{s_{m, \gamma}}$, we have

$$
\sum_{(l, k) \in V_{m}} \mathbb{E}\left(\left(W_{l, k}^{m}\right)^{2}\right) \leq \frac{m(m-1)}{4 \mathbb{E}\left(\mathcal{P}\left(t_{m, \gamma}\right)\right)}\left(a_{1}^{2}+a_{2}^{2}\right) \times 8 p_{t_{m, \gamma}}\left(1-p_{t_{m, \gamma}}\right) \leq a_{1}^{2}+a_{2}^{2} .
$$

Thus, the sum is bounded. Finally, for (iii),

$$
\begin{aligned}
& \operatorname{Var}\left(S_{m}\right)=\operatorname{Var}\left(\sqrt{\frac{1}{4 \mathbb{E}\left(\mathcal{P}\left(t_{m, \gamma}\right)\right)}}\left(a_{1} \mathcal{P}_{m}\left(t_{m, \gamma}\right)+a_{2} \mathcal{P}_{m}\left(s_{m, \gamma}\right)\right)\right) \\
& =\frac{1}{4 \mathbb{E}\left(\mathcal{P}\left(t_{m, \gamma}\right)\right)}\left(a_{1}^{2} \operatorname{Var}\left(\mathcal{P}\left(t_{m, \gamma}\right)\right)+2 a_{1} a_{2} \operatorname{Cov}\left(\mathcal{P}\left(t_{m, \gamma}\right), \mathcal{P}\left(s_{m, \gamma}\right)\right)+a_{2}^{2} \operatorname{Var}\left(\mathcal{P}\left(t_{m, \gamma}\right)\right)\right) \\
& =\frac{\left(a_{1}^{2}+a_{2}^{2}\right)}{4 \mathbb{E}\left(\mathcal{P}\left(t_{m, \gamma}\right)\right)} \operatorname{Var}\left(\mathcal{P}\left(t_{m, \gamma}\right)\right)+\frac{1}{4 m(m-1)} \frac{2 a_{1} a_{2}}{4 p_{t_{m, \gamma}}\left(1-p_{t_{m, \gamma}}\right)} \operatorname{Cov}\left(\mathcal{P}\left(t_{m, \gamma}\right), \mathcal{P}\left(s_{m, \gamma}\right)\right) .
\end{aligned}
$$

Note that $s_{m, \gamma}>t_{m, \gamma}$, thus, by Proposition 3.2,

$$
\begin{aligned}
\operatorname{Cov}\left(\mathcal{P}\left(t_{m, \gamma}\right), \mathcal{P}\left(s_{m, \gamma}\right)\right)=4 p_{s_{m, \gamma}}(1- & \left.p_{t_{m, \gamma}}\right)\left(\left(4 m^{2}-7 m+2\right)\right. \\
& -2 p_{t_{m, \gamma}}\left(1-p_{s_{m, \gamma}}\right)\left(7 m^{2}-13 m+4\right) \\
& \left.+2\left(p_{t_{m, \gamma}}-p_{s_{m, \gamma}}\right)\left(3 m^{2}-6 m+2\right)\right) .
\end{aligned}
$$

Given that $p_{t_{m, \gamma}}=1-p_{s_{m, \gamma}}$, we have

$\operatorname{Cov}\left(\mathcal{P}\left(t_{m, \gamma}\right), \mathcal{P}\left(s_{m, \gamma}\right)\right)=4\left(1-p_{t_{m, \gamma}}\right)^{2}\left(\left(4 m^{2}-7 m+2\right)\right.$ 


$$
\left.-2 p_{t_{m, \gamma}}^{2}\left(7 m^{2}-13 m+4\right)+2\left(2 p_{t_{m, \gamma}}-1\right)\left(3 m^{2}-6 m+2\right)\right) .
$$

Recall that, $p_{t_{m, \gamma}} \rightarrow 1$, thus, $\frac{\operatorname{Cov}\left(\mathcal{P}\left(t_{m, \gamma}\right), \mathcal{P}\left(s_{m, \gamma}\right)\right)}{m(m-1) p_{t_{m, \gamma}}\left(1-p_{t_{m, \gamma}}\right)} \underset{m \rightarrow \infty}{\longrightarrow} 0$. Hence, from Equation (25), we get that $\operatorname{Var}\left(S_{m}\right) \underset{m \rightarrow \infty}{\longrightarrow} a_{1}^{2}+a_{2}^{2}$, since $\frac{\operatorname{Var}\left(\mathcal{P}\left(t_{m, \gamma}\right)\right.}{\mathbb{E}\left(\mathcal{P}\left(t_{m, \gamma}\right)\right.} \underset{m \rightarrow \infty}{\longrightarrow} 4$ by Lemma 6.1 .

\section{Appendix A: Numerical illustrations of Theorem 3.3}

In order to illustrate the multivariate CLT of Theorem 3.3, we follow the example given by [14]. We start by simulating a sample of 900 images of size $1000 \times$ 1000 pixels generated from a Uniform distribution and compute the perimeter without the border using the Algorithm 1 associated to the binary image for a threshold $t=0.5$. We create the histogram of the variable:

$$
A_{m}(t)=\frac{m\left(\check{\mathcal{P}}_{m}(t)-\mathbb{E}\left(\check{\mathcal{P}}_{m}(t)\right)\right)}{\sqrt{\sigma^{\star 2}(t)}}
$$

and compare it to the plot of Gaussian density. To illustrate the bivariate CLT, let $s=0.6$ and $t=0.2$, we use the Python function stats.normaltest from scipy's library to do a normal test based on [10]'s method. This function tests whether a sample differs from a normal distribution. Thus, we plot the $p$-value of the test conducted on the projection $\cos (\theta) A_{m}(t)+\sin (\theta) A_{m}(s)$ for $\theta \in$ $[0,2 \pi)$. This method mimics the Cramèr-Wold Theorem 7.1. We also use the Mahalanobis distance to show the joint Gaussianity. This illustration is based on the fact that if $\mathbf{X} \sim \mathcal{N}_{d}(\mu, \Sigma)$ then, the Mahalanobis distance $D^{2}$ with entries $D_{i}^{2}$

$$
D_{i}^{2}=\left(X_{i}-\mu\right)^{\prime} \Sigma^{-1}\left(X_{i}-\mu\right), i=1, \ldots, m^{2}
$$

is distributed according to a $\chi_{d^{2}}^{2}$ distribution with $d$ degrees of freedom. Using the theoretical value of $\Sigma^{\star}$ and numerically estimating the sample average $\mu$, we compute the value $D_{i}^{2}$ and compare it with a $\chi_{d}^{2}$-distribution, using the QQ-plot method. In Figure 10, we gathered the obtained results from these numerical studies which confirm the joint normal behaviour of $\left(A_{m}(t), A_{m}(s)\right)$.

\section{Appendix B: Comparison between $\sigma^{2}(s)$ and $\sigma_{\mathrm{k}-\mathrm{s}}^{2}(s)$ variances}

Figure 11 below provides a comparison between the two variance values $\sigma^{2}(s)$ in (7) (red full line) and $\left(\sigma_{\mathrm{k}-\mathrm{s}}\right)^{2}(s)$ in (14) (black dashed line), for $p_{s} \in(0,0.5)$. As notice in Remark 4, Figure 11 shows that the variance of the ratio computed using the perimeter is lower than the variance of the ratio computed using a Kolmogorov Smirnov method. 

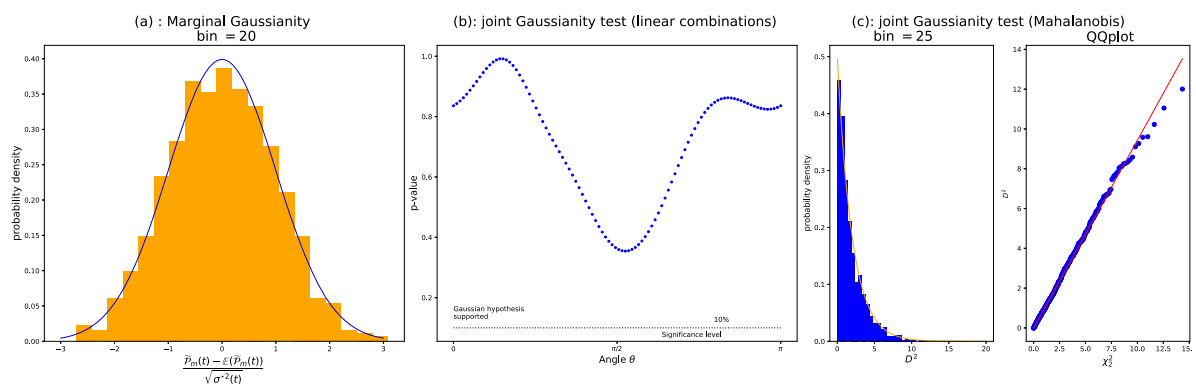

FIG 10. (a.) Univariate Gaussianity of $A_{m}(t)$ in (26) for $m=1200$ and $t=0.5$ for $a$ bin size equal to 20. The solid blue line represents the corresponding standard Gaussian density. We propose two validation methods of the multivariate Gaussianity of the bivariate vector $\left(A_{m}(s), A_{m}(t)\right)$ with $s=0.6$ and $t=0.2$. (b.) $p$-value for $\cos (\theta) A_{m}(t)+\sin (\theta) A_{m}(s)$ for $\theta \in[0,2 \pi)$, computed using the function stats.normaltest from scipy. (c.) Estimated probability density of $D^{2}$ (blue histogram) and theoretical Chi-squared density (red curve). $Q Q$-plot between the theoretically predicted $\chi_{2}^{2}$ quantiles and the empirical quantiles of $D^{2}$.

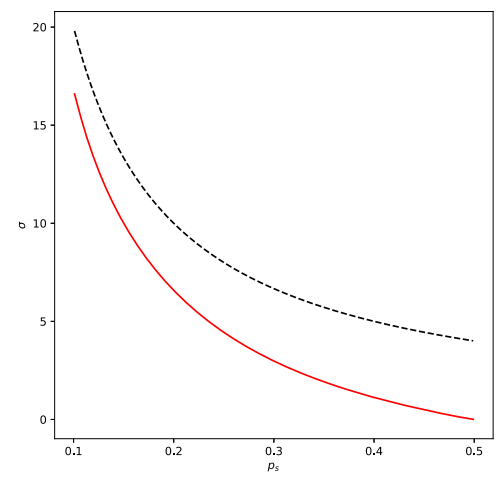

FIG 11. Variance values $\sigma^{2}(s)$ in (7) in (red full line) and $\left(\sigma_{k-s}\right)^{2}(s)$ in (14) (black dashed line $)$, for $p_{s} \in(0.1,0.5)$

\section{Appendix C: Optimization problem for the variance function}

For statistical purposes, it is important to identify the points where the variance function in Equation (4) reaches its maximum and minimum values.

Let us consider the following optimization problem:

$$
\begin{aligned}
p_{t}^{\star} & =\underset{p_{t}}{\arg \min } \sigma_{\tilde{\mathcal{P}}}^{2}\left(p_{t}, m\right) \\
& =\underset{p_{t}}{\arg \min } \frac{4}{m^{4}} p_{t}\left(1-p_{t}\right)\left(\left(4 m^{2}-7 m+2\right)-2 p_{t}\left(1-p_{t}\right)\left(7 m^{2}-13 m+4\right)\right) .
\end{aligned}
$$

By using XCAS, we get that the set of extrema of this variance function is equal to 


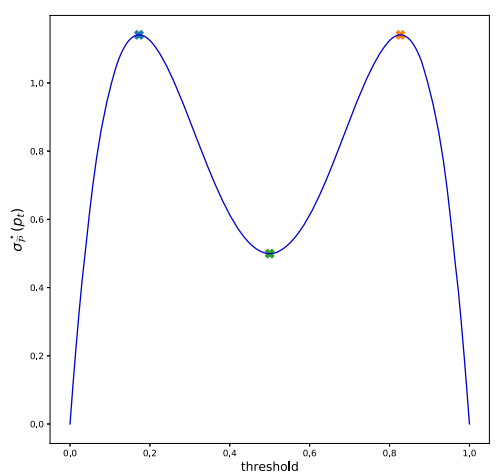

FIG 12. Curve of the variance function in Equation (4) for a Uniform white noise model for $m=1024$, the extrema are reached at $p_{t} \in\{0.172,0.5,0.82\}$.

$$
\begin{gathered}
\left\{\frac{1}{2}-\frac{\sqrt{84 m^{4}-324 m^{3}+416 m^{2}-200 m+32}}{28 m^{2}-52 m+16}, \frac{1}{2}, \frac{1}{2}+\right. \\
\left.\frac{\sqrt{84 m^{4}-324 m^{3}+416 m^{2}-200 m+32}}{28 m^{2}-52 m+16}\right\}:=\left\{\alpha_{1}, \alpha_{2}, \alpha_{3}\right\} .
\end{gathered}
$$

By doing an analytical study, we get the maximum value of the variance is reached on $\alpha_{1}$ and $\alpha_{3}$ and the minimum value for $\alpha_{2}$ (see Figure 12 for an illustration in the Uniform white noise model).

\section{References}

[1] P. A. R. Ade, N. Aghanim, Y. Akrami, P. K. Aluri, M. Arnaud, M. Ashdown, J. Aumont, C. Baccigalupi, A. J. Banday, and et al. Planck 2015 results: XVI. Isotropy and statistics of the CMB. Astronomy $\&$ Astrophysics, 594:A16, 2016.

[2] R. J. Adler. The Geometry of Random Field. John Wiley \& Sons, 1981. MR0611857

[3] J. M. Azaïs and M. Wschebor. Level sets and extrema of random processes and fields. John Wiley \& Sons, 2009. MR2478201

[4] H. Biermé, E. Di Bernardino, C. Duval, and A. Estrade. Lipschitz-Killing curvatures of excursion sets for two-dimensional random fields. Electronic Journal of Statistics, 13(1):536-581, 2019. MR3911693

[5] Hermine Biermé and Agnès Desolneux. The effect of discretization on the mean geometry of a 2d random field. Annales Henri Lebesgue, 2021. MR4108116

[6] P. Billingsley. Probability and Measure (3 ed.). John Wiley and Sons, 1995. MR1324786

[7] C. Butler. A test for symmetry using the sample distribution function. The Annals of Mathematical Statistics, 40(6):2209-2210, 1969. 
[8] S.N. Chiu, D. Stoyan, W.S. Kendall, and J. Mecke. Stochastic Geometry and Its Applications. Wiley Series in Probability and Statistics. Wiley, 2013. MR3236788

[9] S. Csörgő and C. R. Heathcote. Testing for Symmetry. Biometrika, 74(1):177-184, 1987. MR0885930

[10] R. D'Agostino and E. S. Pearson. Tests for departure from normality. Empirical results for the distributions of $b^{2}$ and $\sqrt{b_{1}}$. Biometrika, 60(3):613622, 12 1973. MR0339372

[11] F. Dalmao, J. León, and E. Mordecki. Asymptotic normality of high levellarge time crossings of a Gaussian process. Stochastic Processes and their Applications, 06 2017. MR3926558

[12] A. Desolneux. Stochastic methods for image analysis. In Stochastic geometry, volume 2237 of Lecture Notes in Math., pages 87-127. Springer, Cham, 2019. MR3931584

[13] E. Di Bernardino and C. Duval. Statistics for Gaussian random fields with unknown location and scale using Lipschitz-Killing curvatures. Scandinavian Journal of Statistics, n/a(n/a):1-42, 2020.

[14] E. Di Bernardino, J. León, and T. Tchumatchenko. Cross-Correlations and Joint Gaussianity in Multivariate Level Crossing Models. Journal of mathematical neuroscience, 4:22, 04 2014. MR3197020

[15] Bruno Ebner, Norbert Henze, Michael A. Klatt, and Klaus Mecke. Goodness-of-fit tests for complete spatial randomness based on Minkowski functionals of binary images. Electronic Journal of Statistics, 12(2):2873 2904, 2018. MR3855358

[16] A. Estrade and J. R. León. A central limit theorem for the Euler characteristic of a Gaussian excursion set. The Annals of Probability, 44(6):3849-3878, 2016. MR3572325

[17] G. Flandin and K. J. Friston. Topological inference. In Arthur W. Toga, editor, Brain Mapping: an Encyclopedic Reference, pages 495-500. Academic Press, 2015.

[18] J. R. Gott, D. C. Hambrick, M. S. Vogeley, J. Kim, C. Park, Y-Y. Choi, R. Cen, J. P. Ostriker, and K. Nagamine. Genus Topology of Structure in the Sloan Digital Sky Survey: Model Testing. The Astrophysical Journal, 675(1):16, 2008.

[19] M. K. Gupta. An asymptotically nonparametric test of symmetry. Ann. Math. Statist., 38:849-866, 1967. MR0208761

[20] L. Heinrich. Stable limit theorems for sums of multiply indexed mdependent random variables. Mathematische Nachrichten, 127(1):193-210, 1986. MR0861726

[21] B. Ivanovic, B. Miloševic, and M. Obradovic. Comparison of symmetry tests against some skew-symmetric alternatives in i.i.d. and non-i.i.d. setting. Computational Statistics $\mathscr{E}$ Data Analysis, 151(C), 2020. MR4103316

[22] B. Jähne. Digital Image Processing 6th Edition. Springer, Berlin [u.a.], 2005.

[23] M. Kratz and S. Vadlamani. Central limit theorem for Lipschitz-Killing curvatures of excursion sets of Gaussian random fields. Journal of Theoretical Probability, 2017. MR3842168 
[24] R. Lachièze-Rey. Bicovariograms and Euler characteristic of random fields excursions. Stochastic Processes and their Applications, 129(11):4687-4703, 2019. MR4013877

[25] R. Lachièze-Rey. Normal convergence of nonlocalised geometric functionals and shot-noise excursions. Ann. Appl. Probab., 29(5):2613-2653, 2019. MR4019871

[26] Douglas E. Lake and Daniel M. Keenan. Identifying minefields in clutter via collinearity and regularity detection, volume 2496. SPIE, 1995.

[27] D. Marinucci. Testing for non-Gaussianity on cosmic microwave background radiation: a review. Statist. Sci., 19(2):294-307, 2004. MR2140543

[28] A. Mira. Distribution-free test for symmetry based on Bonferroni's measure. Journal of Applied Statistics, 26, 03 1997. MR1731875

[29] W. K. Pratt. Digital Image Processing. John Wiley \& Sons, Inc., USA, 1978.

[30] J. F. Quessy. On Consistent Nonparametric Statistical Tests of Symmetry Hypotheses. Symmetry, 8, 2016. MR3501189

[31] J. Schmalzing and K. M. Górski. Minkowski functionals used in the morphological analysis of cosmic microwave background anisotropy maps. Monthly Notices of the Royal Astronomical Society, 297(2):355-365, June 1998.

[32] R. Schneider and W. Weil. Stochastic and integral geometry. Probability and its Applications. Springer-Verlag, Berlin, 2008. MR2455326

[33] C. Thäle. 50 years sets with positive reach - a survey. Surveys in Mathematics and its Applications, 3:123-165, 2008. MR2443192

[34] K. J. Worsley. Local maxima and the expected Euler characteristic of excursion sets of $\chi^{2}, F$ and $t$ fields. Advances in Applied Probability, 26(1):13-42, 1994. MR1260300

[35] M. Wschebor. Surfaces aléatoires: mesure géométrique des ensembles de niveau, volume 1147. Springer, 2006. MR0871689 